ESAIM: M2AN 49 (2015) 459-480

DOI: $10.1051 / \mathrm{m} 2 \mathrm{an} / 2014040$
ESAIM: Mathematical Modelling and Numerical Analysis

www.esaim-m2an.org

\title{
REGULARIZED RECURSIVE NEWTON-TYPE METHODS FOR INVERSE SCATTERING PROBLEMS USING MULTIFREQUENCY MEASUREMENTS *, **
}

\author{
Mourad Sini ${ }^{1}$ And Nguyen Trung Thành ${ }^{2}$
}

\begin{abstract}
We are concerned with the reconstruction of a sound-soft obstacle using far field measurements of scattered waves associated with incident plane waves sent from one incident direction but at multiple frequencies. We define, at each frequency, observable shapes as the ones which are described by finitely many modes and produce far field patterns close to the measured one. Our analysis consists of two steps. In the first step, we propose a regularized recursive Newton method for the reconstruction of an observable shape at the highest frequency knowing an estimate of an observable shape at the lowest frequency. We formulate conditions under which an error estimate in terms of the frequency step, the number of Newton iterations, and noise level can be proved. In the second step, we design a multilevel Newton method which has the same accuracy as the one described in the first step but with weaker assumptions on the quality of the estimate of the observable shape at the lowest frequency and a small frequency step (or a large number of Newton iterations). The performances of the proposed algorithms are illustrated with numerical results using simulated data.
\end{abstract}

Mathematics Subject Classification. 35R30, 65N21, 78A46.

Received November 6, 2013. Revised August 26, 2014

Published online March 4, 2015.

\section{INTRODUCTION}

We consider the problem of reconstructing the shape of a two-dimensional (2-d) sound-soft acoustic obstacle using far field measurements associated with incident plane waves sent from only one incident direction but at

Keywords and phrases. Inverse obstacle scattering, multifrequency, convergence, Newton method.

* M. Sini was supported by the Johann Radon Institute for Computational and Applied Mathematics (RICAM), Austrian Academy of Sciences.

** N.T. Thành was supported by US Army Research Laboratory and US Army Research Office Grants W911NF-11-1-0399.

1 Johann Radon Institute for Computational and Applied Mathematics (RICAM), Austrian Academy of Sciences, Altenbergerstrasse 69, 4040 Linz, Austria. mourad.sini@oeaw.ac.at

2 Department of Mathematics \& Statistics, University of North Carolina at Charlotte, 9201 University City Blvd, Charlotte, NC 28223, USA. Current address: Department of Mathematics, Iowa State University, Carver Hall, Ames, IA 500011, USA. thanh@iastate.edu 
multiple frequencies. The forward model can be represented by the following Dirichlet boundary value problem

$$
\begin{aligned}
& \Delta u(x)+k^{2} u(x)=0, x \in \mathbb{R}^{2} \backslash \bar{D}, \\
& u(x)=0, x \in \partial D \\
& \lim _{|x| \rightarrow \infty} \sqrt{|x|}\left[\frac{\partial u^{s}(x)}{\partial|x|}-i k u^{s}(x)\right]=0,
\end{aligned}
$$

where $k$ is the wavenumber, $u$ is the total wave and $u^{s}:=u-u^{i}$ is the scattered wave. Here, $u^{i}$ is the incident plane wave given by $u^{i}(x):=\mathrm{e}^{i k x \cdot d}$ with $d \in \mathbb{S}^{1}:=\left\{x \in \mathbb{R}^{2}:|x|=1\right\}$ being the direction of incidence. The well-posedness of the problems (1.1)-(1.3) for each wavenumber $k$ is well-known under the assumption that $\partial D$ is Lipschitz (see, e.g., [22]). Moreover, we have the following asymptotic behavior of the scattered field $u^{s}$ at infinity

$$
u^{s}(x)=\frac{\mathrm{e}^{i k|x|}}{\sqrt{|x|}} u^{\infty}(\hat{x})+O\left(|x|^{-3 / 2}\right),|x| \rightarrow \infty,
$$

where $\hat{x}:=x /|x|$ and $u^{\infty}$ is an analytic function on $\mathbb{S}^{1}$ referred to as the far field pattern of the scattered wave $u^{s}$.

The inverse problem we investigate here is to reconstruct the obstacle $D$ from measured far field patterns $u^{\infty}(\hat{x}, k), \hat{x} \in \mathbb{S}^{1}$, for one direction of incidence $d \in \mathbb{S}^{1}$ and multiple wavenumbers $k$ in the interval $\left[k_{l}, k_{h}\right](0<$ $\left.k_{l}<k_{h}\right)$. Here we denote the far field pattern by $u^{\infty}(\hat{x}, k)$ to emphasize its dependence on the wavenumber $k$.

Let us recall some known results concerning this inverse problem. It has a unique solution if a band of wavenumbers $\left[k_{l}, k_{h}\right]$ is used, see, e.g., [26]. If the measurements correspond to a finite number of frequencies, as we consider in this paper, then the uniqueness of the solution is guaranteed if the lowest frequency is small enough, see, e.g., [11,14]. For local uniqueness at each frequency, we refer to [29]. If more a priori information about the obstacle's shape is available, then some global uniqueness results at an arbitrary but fixed frequency have been published. For example, if the obstacles are polygonal, see $[1,7]$ and if thes obstacles are nowhere analytic, see [17]. Regarding the stability issue, loglog stability estimates are given in [18] and an improved $\log$ stability estimate is shown in [27]. In the high frequency regime, a conditional asymptotic Hölder stability estimate in the part of the boundary $\partial D$, of a convex obstacle $D$, illuminated by the incident plane wave $u^{i}$ is obtained in [28].

The main advantage of using multifrequency data is that it can help to obtain accurate reconstructions without the need for a good initial guess. Let us explain the reasons why we can expect these two features. The first reason is that at low frequencies only big features of the obstacle are retrievable due to the instability of the original problem. Therefore, we should choose a small number of unknown parameters for representing the obstacle's shape. This reduces the instability of the reconstruction algorithm. The second one is that at high frequencies more details of the obstacle can be reconstructed. However, the objective functional may have many local minima. Using the reconstruction result at a lower frequency helps to avoid getting a false local minimum.

Different reconstruction methods using multifrequency data have been proposed in the last two decades or so. The first type of method is known as frequency-hopping algorithms which use the reconstruction at a frequency as an initial guess at a higher frequency. Several numerical results, using either simulated data, see e.g., $[6,8,28]$, or experimental data, see, e.g., $[4,30,31]$, have been demonstrated. However, convergence of this type of algorithms was only investigated in $[3,28]$ for the so-called recursive linearization algorithm (RLA) proposed in [6]. Another type of methods using multifrequency/multiwaves data, related to the sampling methods, can be found in $[15,16,25]$.

Inspired by the presentation in [6], we define, for each frequency, finite dimensional observable shapes as the ones which can be described by a finite number of parameters and produce far field patterns close to the measured one, see Definition 2.1. Our goal then is to reconstruct an observable shape at the highest available frequency. The link between this observable shape and the true one relates to the stability issue, see [28] and Section 4.3 for more explanations. To achieve this goal, we proceed as follows. 
- First, we propose a regularized projected recursive Newton method for solving this inverse problem, see Section 4. The idea is to use a certain number of Newton iterations at each frequency, starting from the lowest one, and then the reconstruction is used to linearize the problem at the next higher frequency. We prove in Section 4.2 that this algorithm is convergent in the sense that if the reconstructed shape at the lowest frequency is sufficiently close to an observable shape at that frequency, the reconstructed shape at higher frequencies also approximate well a set of corresponding observable shapes. Moreover, the error of the reconstruction of the observable shape at the highest frequency is guaranteed to be arbitrarily small for noise-free data. For noisy data, an additional term of the order $\delta^{2 / 3}$, where $\delta$ is the noise level of the measured data, appears in the error. Note that an observable shape at a high frequency usually approximates the true one better than an observable shape at a low frequency, at least in the part illuminated by the incident wave, see [28]. The result obtained here is a significant improvement compared to the linear convergence rate of the RLA obtained in $[3,28]$.

- Second, a multi-level Newton method is proposed and its convergence is also investigated. The main idea of this method is to divide the whole frequency set into subsets and each of them is treated using the algorithm of Section 4. The difference between these two methods is that in multi-level Newton method the regularization parameter associated with different frequency subsets can be chosen to be different whereas in the algorithm of Section 4 this parameter is fixed at all frequencies. This adaptive choice of the regularization parameter allows us to obtain the same error estimate as the previous algorithm but with less restrictive requirements on the accuracy of the reconstruction at the lowest frequency. This topic is discussed in Section 5 . Related to this approach, we cite the work [12] which also investigates a multi-level projected steepest descent method in Banach spaces in which a discrepancy principle is used for stopping the iterative process at each frequency.

Both of these Newton methods require a good approximation of the observable shape at the lowest frequency. In this work, this first guess is obtained as follows, see Section 3. Using low frequency asymptotic expansions, we prove that at a low enough frequency, the modulus of the far field pattern of the unknown obstacle can be approximated by that of a circle. Then, the radius of this circle is estimated. Using a result of [20], we show that the determination of the radius is equivalent to finding the unique zero of a monotonically increasing function of one variable. Moreover, this unique zero lies in a given interval, whose length is inversely proportional to the frequency. Hence, this zero can be found easily, e.g., by bisection method.

Finally, we show in Section 6 some numerical results using simulated data to demonstrate the performance of the aforementioned algorithms. Our numerical results are consistent with the theoretical analysis.

\section{BOUNDARY-TO-FAR-FIELD OPERATOR AND OBSERVABLE SHAPES}

In this work, we consider the case of star-shaped obstacles whose boundary $\partial D$ can be represented by

$$
\partial D=\left\{x(t) \in \mathbb{R}^{2}: x(t)=x^{0}+r(t)(\cos t, \sin t), t \in[0,2 \pi]\right\},
$$

where $x^{0}$ is a given internal point of $D$ in $\mathbb{R}^{2}$ and the radial function $r$ is positive in $[0,2 \pi]$ with $r(0)=r(2 \pi)$. In the following, we write $D(r)$ to indicate the dependence of the obstacle on its radial function $r$.

For each wavenumber $k$, we define the boundary-to-far field operator (or far field operator, for short) $F(\cdot, k$ ) which maps each radial function $r$ to the far field pattern $u^{\infty}(\cdot, k, r)$ of the forward scattering problems (1.1)(1.3) with $D=D(r)$. In this paper, we assume that the shape is of class $C^{3}$, i.e., the $2 \pi$-periodic extension of the radial function $r$ from $[0,2 \pi]$ to $\mathbb{R}$ belongs to $C^{3}(\mathbb{R})$. This smoothness guarantees the regularity of the derivatives of the far field operator used in Section 4.2. We denote by $X_{a d}$ the set of radial functions of $C^{3}$-class starlike shapes. This set is considered as the admissible set in our algorithm. 
We denote by $\partial_{r} F(r, k)$ the Fréchet derivative of $F$ with respect to the radial function. It was proved in $[19,24]$ that $\partial_{r} F(r, k) a=\tilde{u}^{\infty}$ for $a \in C^{1}[0,2 \pi]$, where $\tilde{u}^{\infty}$ is the far field pattern of the following problem

$$
\begin{aligned}
& \Delta \tilde{u}(x)+k^{2} \tilde{u}(x)=0, x \in \mathbb{R}^{2} \backslash \bar{D}, \\
& \tilde{u}(x(t))=-a(t)(\cos t, \sin t) \cdot \nu(x(t)) \frac{\partial u(x(t))}{\partial \nu}, x(t) \in \partial D, t \in[0,2 \pi], \\
& \lim _{|x| \rightarrow \infty} \sqrt{|x|}\left[\frac{\partial \tilde{u}(x)}{\partial|x|}-i k \tilde{u}(x)\right]=0,
\end{aligned}
$$

with $\nu(x)$ being the outward unit normal vector at $x \in \partial D$.

Let $X$ be a Hilbert space such that $X_{a d} \subset X$ and the Fréchet derivative operator $\partial_{r} F(r, k) a$ is well-defined for all $a \in X$. Since problems $(2.2)-(2.4)$ has a solution even when $\left.a \in L_{2}[0,2 \pi]\right), \partial_{r} F(r, k)$ can be extended to $L_{2}\left(\mathbb{S}^{1}\right)$, see [10]. Therefore, we can choose the space $X$ to be $L_{2}[0,2 \pi]$. However, other Hilbert spaces can be used as well. For generality, in the following we use the notation $X$ instead of $L_{2}$. Note that $\partial_{r} F(r, k)$ is an injective linear operator from $X$ to $L_{2}\left(\mathbb{S}^{1}\right)$ for $r \in X_{a d}$, see $[10,19]$.

In the following Sections, we denote by $u_{m}^{\infty, \delta}(\cdot, k) \in L_{2}\left(\mathbb{S}^{1}\right)$ the noisy measured far field pattern at the wavenumber $k$ with additive random noise of magnitude (noise level) $\delta \geq 0$. We define the operator $\tilde{F}_{\delta}$ from $X_{a d}$ to $L_{2}\left(\mathbb{S}^{1}\right)$ by $\tilde{F}_{\delta}(r, k):=F(r, k)-u_{m}^{\infty, \delta}(\cdot, k)$. The norms in $X$ and $L_{2}\left(\mathbb{S}^{1}\right)$ are denoted by $\|\cdot\|_{X}$ and $\|\cdot\|_{2}$, respectively.

Since the radial function $r(t)$ is $2 \pi$-periodic, it can be represented by the following Fourier series

$$
r(t)=\beta_{0}+\sum_{m=1}^{\infty}\left(\beta_{m} \cos m t+\gamma_{m} \sin m t\right) .
$$

We note that the Fourier coefficients $\beta_{m}$ and $\gamma_{m}$ converge to zero as $m \rightarrow \infty$. Their convergence rate depends on the smoothness of the function $r(t)$, see [13]. For each number $M \in \mathbb{N}$, we define the cut-off approximation $\bar{r}^{M}(t)$ of $r(t)$ by

$$
\bar{r}^{M}(t):=\beta_{0}+\sum_{m=1}^{M}\left(\beta_{m} \cos m t+\gamma_{m} \sin m t\right) .
$$

We denote by $\bar{X}_{M}$ the subspace of $X$ spanned by the basis $\{1, \cos t, \sin t, \ldots, \cos M t, \sin M t\}$ for $M \in \mathbb{N}$. We also denote by $\bar{X}_{M}^{+}:=\left\{\varphi \in \bar{X}_{M}: \varphi(t)>0, \forall t \in[0,2 \pi]\right\}$. Clearly, $\bar{X}_{M}^{+} \subset X_{a d}$.

Let us recall the concept of finite dimensional observable shapes which was defined in [28], see also [6] for a similar concept for penetrable obstacles. For this purpose, we note that for a given wavenumber $k$ and a number $\tau>1$, there exists a number $M_{0}(k) \in \mathbb{N}$ depending on $k$ such that $\left\|F\left(\bar{r}^{M}, k\right)-F(r, k)\right\|_{2} \leq(\tau-1) \delta$ for all $M \geq M_{0}(k)$. Consequently, $\left\|F\left(\bar{r}^{M}, k\right)-u_{m}^{\infty, \delta}(\cdot, k)\right\|_{2} \leq \tau \delta$ for $M \geq M_{0}(k)$. Note that $M_{0}(k)$ also depends on $\tau$ and $\delta$, but we ignore these parameters since they are fixed throughout the paper.

Definition 2.1. For each wavenumber $k$ and a given value $\tau>1$, a finite dimensional observable shape (or, for short, observable shape), denoted by $D(\tilde{r}(k))$, is defined as a start-like shape whose radial function $\tilde{r}(k)$ contains only a finite number of Fourier modes and the corresponding far field pattern $F(\tilde{r}(k), k)$ satisfies the condition $\left\|F(\tilde{r}(k), k)-u_{m}^{\infty, \delta}(\cdot, k)\right\|_{2} \leq \tau \delta$.

By this definition, an observable shape is a shape which basically produces the same measured data as the true one (up to the noise level) but consists of only a finite number of Fourier modes. As pointed out above, there always exist observable shapes at each frequency. For example, $D\left(\bar{r}^{M}\right)$ is an observable shape of the obstacle $D$ for $M$ large enough. Moreover, there are infinitely many observable shapes at each frequency. In addition, an observable shape may be quite different from the true shape due to the ill-posedness of the inverse problem under investigation. The question on how these observable shapes approximate the true one relates directly to the stability of the inverse problem. As discussed in [28], an observable shape, roughly speaking, approximates 
"big" features of the true shape at low frequencies while it is close to the true shape in the part illuminated by the incident plane wave at high frequencies.

In the last inequality of Definition 2.1 we made use of the value $\tau \delta$ instead of $\delta$ because if the latter is used, the finite dimensional observable shapes might not exist. However, it is possible to choose $\tau$ such that it is just slightly larger than 1 while $M_{0}(k)$ can still be chosen not too large. This can be explained using the Heisenberg's uncertainty principle in Physics on the resolution limit of scattering problems. It says that, at a fix frequency, we cannot observe small details of the scatterer using noisy measurements of the far field pattern, even for a small noise level. Therefore, choosing many Fourier modes may not help to improve the reconstruction accuracy but increases the instability of inverse algorithms. Therefore, $M_{0}(k)$ should not be chosen too large. As shown in [6], this resolution limit is about half of the wavelength for weak penetrable scatterers, see also $[2,3]$.

In the following, we simplify the inverse problem by determining a set of observable shapes instead of the true one. By this simplification, the inverse problem becomes finite dimensional.

\section{Determining an APProximating Circle at The loweSt FREQUenCy}

As demonstrated in theoretical analysis in Section 4.2 and numerical results in Section 6, the accuracy of the reconstructed shape at the highest frequency depends on the accuracy of the reconstruction of an observable shape at the lowest frequency, which serves as the first guess for the Newton methods.

One way to obtain this first guess is by minimizing a least-squares objective functional at $k=k_{0}$. If $k_{0}$ is chosen small, we can choose a subspace $X_{0}$ such that it contains only a small number of degrees of freedom and find an approximation of the shape in this subspace. In this case, the minimization problem is expected to be stable and its objective functional is usually convex in a large domain. That means, a good initial guess may not be needed.

In particular, at a sufficiently small wavenumber $k_{0}$, the modulus of the far field pattern of the unknown obstacle can be approximated by that of a circle. Indeed, if $r(t)=\rho r^{*}(t), t \in[0,2 \pi)$, where $\rho>0$ and $r^{*}$ is a fixed radial function which describes the relative shape $B$ of the obstacle, then in the proof of Theorem 3.1 of [15], it was shown that when $k_{0} \rho \ll 1$, the following asymptotic expansion holds:

$$
\sqrt{k_{0}} u^{\infty}\left(\hat{x}, k_{0}\right)=\frac{i \mathrm{e}^{i \pi / 4} C_{B}}{\sqrt{8 \pi} \ln \left(k_{0} \rho\right)} \mathrm{e}^{i k_{0}(d-\hat{x}) \cdot x^{0}}+O\left(\frac{1}{\left(\ln \left(k_{0} \rho\right)\right)^{2}}\right)
$$

for a constant $C_{B}$ depending on $B$. In particular, if $B$ is a circle of radius 1 , we have $C_{B}=2 \pi / i$. On the other hand, for given $\rho$ and $B$ with $k_{0} \rho<1$, there exists a number $r_{0}>0$ such that

$$
\frac{2 \pi}{\left|\ln \left(k_{0} r_{0}\right)\right|}=\frac{\left|C_{B}\right|}{\left|\ln \left(k_{0} \rho\right)\right|} .
$$

That is, the modulus of the far field pattern of the circle $\mathbb{S}\left(x^{0}, r_{0}\right):=\left\{x \in \mathbb{R}^{2}:\left|x-x^{0}\right|=r_{0}\right\}$ has the same dominant term as that of the unknown obstacle in the asymptotic expansion (3.1).

Now we propose a method for obtaining an approximation of this radius by solving a simple 1-d minimization problem. For this purpose, we consider a single datum at only one observation direction $\hat{x}=d$. Denote by $u_{c}^{\infty}\left(\cdot, a, k_{0}\right)$ the far field pattern of the a circle of radius $a$ at wavenumber $k_{0}$. Note that the modulus of a circle does not depend on its location. Consequently, we can choose the center of this circle at the origin. Then, the far field pattern is given by (see e.g., $[9,21]$ )

$$
u_{c}^{\infty}\left(\varphi, a, k_{0}\right)=\mathrm{e}^{-i \pi / 4} \sqrt{\frac{2}{\pi k_{0}}} \sum_{n=-\infty}^{\infty} \frac{J_{n}\left(k_{0} a\right)}{H_{n}^{(1)}\left(k_{0} a\right)} \mathrm{e}^{i n(\varphi-\theta)},
$$

where $J_{n}$ is the Bessel function of order $n, H_{n}^{(1)}$ is the Hankel function of the first kind of order $n$, and $\varphi$ and $\theta$ are respectively the observation and incident angles. The radius $r_{0}$ is then approximated by a zero of the 
following function

$$
\mathcal{F}_{1}(a):=\sqrt{k_{0}}\left[\left|u_{c}^{\infty}\left(d, a, k_{0}\right)\right|-\left|u_{m}^{\infty, \delta}\left(d, k_{0}\right)\right|\right] .
$$

It was proved in $[20]$ that $\left|u_{c}^{\infty}\left(d, a, k_{0}\right)\right|$ is monotonically increasing with respect to $a$ in the interval $\left(0, C / k_{0}\right)$, where $C \approx 0.2303$. Therefore, $\mathcal{F}_{1}$ has at most one zero in this interval. Furthermore, assume that the unknown obstacle $D$ and the approximating circle lie in the disk $B\left(x^{0}, R\right):=\left\{x \in \mathbb{R}^{2}:\left|x-x^{0}\right| \leq R\right\}$, for a given $R>0$. Then, we can choose a small $k_{0}$ so that $C / k_{0}>R$. Then if a zero of $\mathcal{F}_{1}$ exists in $\left(0, C / k_{0}\right)$, it should approximate the radius $r_{0}$ of the approximating circle $\mathbb{S}\left(x^{0}, r_{0}\right)$ for $k_{0}$ small enough since $\left|u_{m}^{\infty, \delta}\left(d, k_{0}\right)\right|$ is approximately equal to $\left|u_{c}^{\infty, \delta}\left(d, r_{0}, k_{0}\right)\right|$ for $k_{0}$ small.

Now we show that there exists a unique zero of $\mathcal{F}_{1}$ in $\left(0, C / k_{0}\right)$. Indeed, (3.1) implies that for a given obstacle, $\sqrt{k_{0}} u^{\infty}\left(d, k_{0}\right) \rightarrow 0$ as $k_{0} \rightarrow 0$. On the other hand, it follows from $(3.3)$ that $\sqrt{k_{0}} u_{c}^{\infty}\left(d, C / k_{0}, k_{0}\right)$ is a constant independent of $k_{0}$. From these properties, we deduce that for a given $\delta$, there exist a small enough wavenumber $k_{0}$ such that

$$
\sqrt{k_{0}}\left|u_{m}^{\infty, \delta}\left(d, k_{0}\right)\right| \leq \sqrt{k_{0}}\left|u_{c}^{\infty}\left(d, C / k_{0}, k_{0}\right)\right|,
$$

It also follows from (3.1) that for a given small $k_{0}, \sqrt{k_{0}} u_{c}^{\infty}\left(d, a, k_{0}\right) \rightarrow 0$ as $a \rightarrow 0$. Hence, there exists $a_{l}>0$ depending on $k_{0}$ such that

$$
\sqrt{k_{0}}\left|u_{c}^{\infty}\left(d, a_{l}, k_{0}\right)\right| \leq \sqrt{k_{0}}\left|u_{m}^{\infty, \delta}\left(d, k_{0}\right)\right| .
$$

From $(3.5)$ and (3.6) it is clear that $\mathcal{F}_{1}(a)$ has a unique zero in the interval $\left(a_{l}, C / k_{0}\right)$. We denote this again by $r_{0}$. This radius is considered as the first guess in Algorithms 4.1 and 5.1.

The determination of this unique zero is simple. For example, we can use the bisection method since we know its lower and upper bounds. Note that no initial guess is required.

\section{A Regularized projected Recursive Newton method}

\subsection{Description of the algorithm}

Suppose that the far field pattern is measured at a set of wavenumbers $k_{n}:=k_{l}+n \Delta k, n=0,1, \ldots, N$, with $\Delta k=\frac{k_{h}-k_{l}}{N}$. Consider a set of increasing finite dimensional subspaces $X_{0} \subseteq X_{1} \subseteq \cdots \subseteq X_{N}$ of $X$. Since elements of $X_{N}$ are smooth, it is clear that $X_{N}^{+} \subset X_{a d}$. Note that, for generality, these subspaces may be different from the subspaces $\bar{X}_{M}$ defined in the previous Section. Assume that we have already obtained an approximation $r_{0} \in X_{0}^{+}$of the radial function at the lowest wavenumber $k_{0}=k_{l}$, see Section 3. Given an integer $J>0$ and an approximation $r_{n} \in X_{n}^{+}$of the radial function at wavenumber $k_{n}$, we denote by $r_{n+1}^{0}:=r_{n}$ and consider $J$ Newton iterations at wavenumber $k_{n+1}$ as follows: $r_{n+1}^{j+1}:=r_{n+1}^{j}+\Delta r_{n+1}^{j}, j=0, \ldots, J-1$, with $\Delta r_{n+1}^{j} \in X_{n+1}$ being the solution of the regularized least-squares minimization problem

$$
\Delta r_{n+1}^{j}:=\operatorname{argmin}_{\Delta r \in X_{n+1}}\left\{\frac{1}{2}\left\|\tilde{F}_{\delta}\left(r_{n+1}^{j}, k_{n+1}\right)+A_{n+1}^{j} \Delta r\right\|_{2}^{2}+\frac{1}{2} \alpha\|\Delta r\|_{X_{n+1}}^{2}\right\}
$$

where $A_{n+1}^{j}$ is the restriction of $\partial_{r} F\left(r_{n+1}^{j}, k_{n+1}\right)$ on the subspace $X_{n+1}$, i.e., $A_{n+1}^{j}:=\left.\partial_{r} F\left(r_{n+1}^{j}, k_{n+1}\right)\right|_{X_{n+1}}$ and $\alpha>0$. The solution to (4.1) is given by

$$
\Delta r_{n+1}^{j}=-R_{n+1}^{j} \tilde{F}_{\delta}\left(r_{n+1}^{j}, k_{n+1}\right)
$$

where $R_{n+1}^{j}:=\left[\alpha I+\left(A_{n+1}^{j}\right)^{*} A_{n+1}^{j}\right]^{-1}\left(A_{n+1}^{j}\right)^{*}$ and $\left(A_{n+1}^{j}\right)^{*}$ is the adjoint operator of $A_{n+1}^{j}$. Hence,

$$
r_{n+1}^{j+1}:=r_{n+1}^{j}-R_{n+1}^{j} \tilde{F}_{\delta}\left(r_{n+1}^{j}, k_{n+1}\right), j=0, \ldots, J-1,
$$

Since $r_{n+1}^{0}=r_{n} \in X_{n}^{+} \subseteq X_{n+1}^{+}$, the approximations $r_{n+1}^{j}$ also belong to the subspace $X_{n+1}$ for $j=1, \ldots, J$. In Theorems 4.5 and 4.6 below we will prove that $r_{n+1}^{j} \in X_{n+1}^{+}$. We choose $r_{n+1}:=r_{n+1}^{J} \in X_{n+1}^{+}$as the reconstruction at the wavenumber $k_{n+1}$. This process is repeated until the highest wavenumber $k_{N}=k_{h}$. The algorithm is summarized as follows. 


\section{Algorithm 4.1.}

- Given measured data $u_{m}^{\infty, \delta}\left(\cdot, k_{n}\right)$ at the wavenumbers $k_{n}, n=0, \ldots, N$, and the parameter $\alpha>0$.

- Step 1: At the lowest wavenumber $k=k_{0}$, choose a subspace $X_{0}$ of $X$ and find an approximation $r_{0} \in X_{0}^{+}$.

- Step 2 (recurrence) For $n=0, \ldots, N-1$

- Choose a subspace $X_{n+1}$ such that $X_{n} \subseteq X_{n+1}$.

- Set $r_{n+1}^{0}:=r_{n}$.

- Compute $r_{n+1}^{j+1}:=r_{n+1}^{j}-R_{n+1}^{j} \tilde{F}_{\delta}\left(r_{n+1}^{j}, k_{n+1}\right)$ for $j=0, \ldots, J-1$.

- Set $r_{n+1}:=r_{n+1}^{J}$.

End (for $n$ ).

\section{Remark 4.2.}

1. In the recursive linearization algorithm, as discussed in $[3,6,28]$, only one Newton step is used at each frequency. In other words, the reconstruction at $k_{n+1}$ is chosen by $r_{n+1}:=r_{n+1}^{1}$.

2. The stopping criterion for Algorithm 4.1 relates to a trade-off between the frequency step $\Delta k$ (or the number of frequencies $N)$ and the number of Newton iterations $J$ to achieve a final error of the order $O\left(\delta^{\frac{2}{3}}\right)$, see Remark 4.7.

Remark 4.3. In order to make Algorithm 4.1 stable, the subspaces $X_{n}, n=0, \ldots, N$, should be chosen such that they are gradually increasing. Indeed, as we mentioned at the end of Section 2 , for a small $k_{n}$ we should not expect to reconstruct more than a few number of Fourier modes. That is, the singular values of $\partial_{r} F\left(r, k_{n}\right)$ are small except the first few ones, see also [28] for a numerical demonstration. As a result, the subspace $X_{n}$ should not be chosen too large since otherwise the inversion procedure will be unstable. Moreover, choosing a large number of Fourier modes may not help to increase the accuracy anyhow. The higher the frequency, the more number of Fourier modes can be expected to be stably reconstructed. That means, the larger the subspace can be chosen.

The effect of the choice of the subspaces $X_{n}$ can also be seen in our convergence analysis in Theorems 4.5 and 4.6. Indeed, the regularization parameter $\alpha$ should be bounded above by the smallest singular value $\sigma$, see (4.5) and (4.13). This singular value $\sigma$ again depends on the dimensions of the subspaces $X_{n}, n=0, \ldots, N$.

\subsection{Convergence of Algorithm 4.1 and error estimates}

In order to prove that the reconstructed shapes obtained by Algorithm 4.1 are good approximations of the corresponding observable shapes, we consider a particular set of the subspaces $X_{n}, n=0, \ldots, N$. More precisely, at each wavenumber $k_{n}$ there exists a number $M_{n} \in \mathbb{N}$ such that the subspace $\bar{X}_{M_{n}}$ defined in Section 2 contains at least one observable shape. In this Section, we assume that $X_{n}, n=0, \ldots, N$, in Algorithm 4.1 are chosen such that $X_{n}=\bar{X}_{M_{n}}$.

For this set of the subspaces $X_{n}$, we assume that there exists a set of observable shapes $D\left(\tilde{r}\left(k_{n}\right)\right), n=0, \ldots, N$, whose radial functions $\tilde{r}\left(k_{n}\right)$ satisfy the following assumptions.

Assumption 1: The radial functions $\tilde{r}\left(k_{n}\right), n=0, \ldots, N$, are bounded from below, i.e., there exists a constant $\tilde{c}>0$ such that

$$
\tilde{c} \leq\left\|\tilde{r}\left(k_{n}\right)\right\|, \text { for all } n,
$$

where $\|\cdot\|$ represents the maximum norm. Since the observable shapes, roughly speaking, are approximations of the true one, this assumption requires that the size of the true obstacle is not too small. As indicated in Theorem 4.5, this lower bound $\tilde{c}$ can be chosen comparable to the regularization parameter $\alpha$, see (4.14), which is reasonably small. That means, this assumption is not very restrictive.

Assumption 2: There exists a constant $d_{0} \geq 1$ such that

$$
\left\|\tilde{r}\left(k_{n+1}\right)-\tilde{r}\left(k_{n}\right)\right\|_{X} \leq d_{0}\left|k_{n+1}-k_{n}\right|, \forall n=0, \ldots, N-1 .
$$


Roughly speaking, this assumption says that there exists a set of observable shapes such that the corresponding radial functions $\tilde{r}\left(k_{n}\right)$ are close for two close wavenumbers. In general, this assumption should not hold for an arbitrary set of observable shapes due to the instable nature of the inverse problem. Moreover, the constant $d_{0}$ may depend on the frequency step. However, by assuming that (4.7) holds, we keep in mind that what we can expect to reconstruct using Algorithm 4.1 is a set of observable shapes which is stably varying from one frequency to the next one. Moreover, the error estimates depend on $d_{0}$. For more detail about the validity of Assumption 2, see Remark 4 of [28].

We denote by $\tilde{A}_{n}:=\left.\partial_{r} F\left(\tilde{r}\left(k_{n}\right), k_{n}\right)\right|_{X_{n}}, n=0,1, \ldots, N$ and $\sigma_{n}$ the smallest singular value of $\tilde{A}_{n}, n=$ $0, \ldots, N-1$. Since these operators are injective, we have $\sigma_{n}>0, n=0, \ldots, N-1$. Finally we define

$$
\sigma:=\min \left\{\sigma_{0}, \ldots, \sigma_{N-1}\right\} .
$$

For the radial functions $\tilde{r}(k), k \in\left[k_{l}, k_{h}\right]$, associated with a given set of observable shapes of $r$, we write the operator $\tilde{F}_{\delta}$ as

$$
\tilde{F}_{\delta}(r, k)=\tilde{F}(r, k)+f^{\delta}(\tilde{r}(k), k)
$$

with $\tilde{F}(r, k):=F(r, k)-F(\tilde{r}(k), k)$ and $f^{\delta}(\tilde{r}(k), k):=F(\tilde{r}(k), k)-u_{m}^{\infty, \delta}(\cdot, k)$. Note that $\left\|f^{\delta}(\tilde{r}(k), k)\right\|_{2} \leq \tau \delta$. It is obvious that

$$
\tilde{F}(\tilde{r}(k), k)=0, \forall k \in\left[k_{l}, k_{h}\right] .
$$

Note that $F(r, k)$ is twice continuously differentiable (see Remark 1 of [28]). Therefore, there exist some positive constants $d_{i}, i=1, \ldots, 4$, such that for all $\|r\| \leq 2 R$, with $R$ defined in Section 3 , and $k \in\left[k_{l}, k_{h}\right]$, we have

$$
\begin{aligned}
&\left\|\partial_{r} \tilde{F}(r, k)\right\|_{\mathcal{L}(X, Y)} \leq d_{1},\left\|\partial_{k} \tilde{F}(r, k)\right\|_{2} \leq d_{2}, \\
&\left\|\partial_{r r}^{2} \tilde{F}(r, k)\right\|_{\mathcal{L}(X \times X, Y)} \leq d_{3},\left\|\partial_{k r}^{2} \tilde{F}(r, k)\right\|_{\mathcal{L}(X, Y)} \leq d_{4} .
\end{aligned}
$$

In this Section, we need the following estimates concerning compact linear operators.

Lemma 4.4. Let $A$ be a compact linear operator from a Hilbert space $X$ to a Hilbert space $Y$ and $R_{\alpha}(A):=$ $\left(\alpha I+A^{*} A\right)^{-1} A^{*}$ with $\alpha>0$. Then

$$
\begin{aligned}
\left\|\left(\alpha I+A^{*} A\right)^{-1}\right\|_{\mathcal{L}(X, X)} & \leq \frac{1}{\alpha} \\
\left\|R_{\alpha}(A)\right\|_{\mathcal{L}(Y, X)} & \leq \frac{1}{2 \sqrt{\alpha}}, \\
\left\|R_{\alpha}(A) A\right\|_{\mathcal{L}(X, X)} & \leq 1
\end{aligned}
$$

Moreover, if $\tilde{A}$ is also a compact linear operator from $X$ to $Y$, we have

$$
\left\|R_{\alpha}(A)-R_{\alpha}(\tilde{A})\right\|_{\mathcal{L}(Y, X)} \leq \frac{9}{4 \alpha}\|A-\tilde{A}\|_{\mathcal{L}(X, Y)} .
$$

We first prove the following result for the case of noiseless data.

Theorem 4.5. Assume that the radial functions $\tilde{r}\left(k_{n}\right), n=0, \ldots, N$, of the observable shapes satisfy Assumptions 1 and 2. Let $X_{n}, n=0, \ldots, N$, be the subspaces $\bar{X}_{M_{n}}$ of $X$ containing these radial functions. Let $r_{n}, n=0, \ldots, N$, be given by Algorithm 4.1 with $\tilde{F}_{\delta}$ being replaced by $\tilde{F}$. Then for a fixed positive real number $\epsilon, 0<\epsilon<3 /\left(2+d_{0}\right)$, and for the regularization parameter $\alpha$ satisfying

$$
\alpha \leq \frac{\epsilon \sigma^{2}}{3-\epsilon}
$$

there exists an integer $N_{0}$ depending on $\epsilon$ and $\alpha$ such that if

$$
\left\|\tilde{r}\left(k_{l}\right)-r_{0}\right\|_{X_{0}} \leq d_{0} c_{0} \alpha<\tilde{c}
$$


with

$$
c_{0}:=\frac{4 \epsilon}{3 d_{3}\left(9 d_{1}+\sqrt{\alpha}\right)},
$$

then we have $r_{n}(t)>0 \forall t \in[0,2 \pi]$ and the following error estimate holds true

$$
\left\|\tilde{r}\left(k_{h}\right)-r_{N}\right\|_{X}=\left\|\tilde{r}\left(k_{h}\right)-r_{N}\right\|_{X_{N}} \leq C_{1}\left(\frac{\epsilon\left(1+d_{0}\right)}{3}\right)^{J-1} \sqrt{\Delta k}, \forall N \geq N_{0},
$$

where $C_{1}$ is a constant independent of $\alpha$ and $N$.

Proof. For $n=0, \ldots, N$ and $j=0, \ldots, J$, we define $e_{n}:=\tilde{r}\left(k_{n}\right)-r_{n}$ and $\tilde{R}_{n}:=\left(\alpha I+\tilde{A}_{n}^{*} \tilde{A}_{n}\right)^{-1} \tilde{A}_{n}^{*}$. We also define $e_{n+1}^{j}:=\tilde{r}\left(k_{n+1}\right)-r_{n+1}^{j}$ for $j=1, \ldots, J ; n=1, \ldots, N$.

We first estimate $\mathrm{e}_{n+1}^{1}$. Here we repeat some arguments of $[3,28]$. It follows from $(4.2)$ that

$$
\begin{aligned}
e_{n+1}^{1} & =\tilde{r}\left(k_{n+1}\right)-r_{n+1}^{0}+R_{n+1}^{0} \tilde{F}\left(r_{n+1}^{0}, k_{n+1}\right) \\
& =\tilde{r}\left(k_{n+1}\right)-\tilde{r}\left(k_{n}\right)+e_{n}-\tilde{R}_{n} \tilde{A}_{n} e_{n}+\tilde{R}_{n} \tilde{A}_{n} e_{n}+R_{n+1}^{0} \tilde{F}\left(r_{n+1}^{0}, k_{n+1}\right) .
\end{aligned}
$$

Hence,

$$
\begin{aligned}
\left\|\mathrm{e}_{n+1}^{1}\right\|_{X_{n+1}} \leq & \left\|\tilde{r}\left(k_{n+1}\right)-\tilde{r}\left(k_{n}\right)\right\|_{X_{n+1}}+\left\|e_{n}-\tilde{R}_{n} \tilde{A}_{n} e_{n}\right\|_{X_{n+1}} \\
& +\left\|\tilde{R}_{n} \tilde{A}_{n} e_{n}+R_{n+1}^{0} \tilde{F}\left(r_{n+1}^{0}, k_{n+1}\right)\right\|_{X_{n+1}} .
\end{aligned}
$$

We recall that $r_{n+1}^{0}=r_{n}$. Let us evaluate the right hand side. Firstly, (4.4) implies that

$$
\left\|\tilde{r}\left(k_{n+1}\right)-\tilde{r}\left(k_{n}\right)\right\|_{X_{n+1}} \leq d_{0} \Delta k
$$

Secondly, from the spectral theory, with the note that $e_{n} \in X_{n}$, we obtain

$$
\left\|e_{n}-\tilde{R}_{n} \tilde{A}_{n} e_{n}\right\|_{X_{n+1}}=\left\|e_{n}-\tilde{R}_{n} \tilde{A}_{n} e_{n}\right\|_{X_{n}} \leq \frac{\alpha}{\alpha+\sigma^{2}}\left\|e_{n}\right\|_{X_{n}}
$$

Thirdly,

$$
\begin{aligned}
\tilde{R}_{n} \tilde{A}_{n} e_{n}+R_{n+1}^{0} \tilde{F}\left(r_{n+1}^{0}, k_{n+1}\right)= & \tilde{R}_{n}\left[\tilde{A}_{n} e_{n}+\tilde{F}\left(r_{n}, k_{n}\right)\right]-\left(\tilde{R}_{n}-R_{n+1}^{0}\right) \tilde{F}\left(r_{n}, k_{n}\right) \\
& +R_{n+1}^{0}\left[\tilde{F}\left(r_{n}, k_{n+1}\right)-\tilde{F}\left(r_{n}, k_{n}\right)\right] .
\end{aligned}
$$

Since $e_{n} \in X_{n}$, we have $\tilde{A}_{n} e_{n}=\partial_{r} F\left(\tilde{r}\left(k_{n}\right), k_{n}\right) e_{n}$. Using the second order Taylor expansion of $\tilde{F}\left(r_{n}, k_{n}\right)$ at $\tilde{r}\left(k_{n}\right),(4.10)$ and $(4.7)-(4.8)$, we obtain

$$
\left\|\tilde{R}_{n}\left[\tilde{A}_{n} e_{n}+\tilde{F}\left(r_{n}, k_{n}\right)\right]\right\|_{X_{n+1}} \leq \frac{d_{3}}{4 \sqrt{\alpha}}\left\|e_{n}\right\|_{X_{n+1}}^{2}=\frac{d_{3}}{4 \sqrt{\alpha}}\left\|e_{n}\right\|_{X_{n}}^{2} .
$$

On the other hand, it follows from Lemma 4.4 and (4.7)-(4.8) that

$$
\begin{aligned}
\left\|\left(\tilde{R}_{n}-R_{n+1}^{0}\right) \tilde{F}\left(r_{n}, k_{n}\right)\right\|_{X_{n+1}} & \leq \frac{9}{4 \alpha}\left\|A_{n+1}^{0}-\tilde{A}_{n}\right\|_{\mathcal{L}\left(X_{n+1}, Y\right)}\left\|\tilde{F}\left(r_{n}, k_{n}\right)-\tilde{F}\left(\tilde{r}\left(k_{n}\right), k_{n}\right)\right\|_{2} \\
& \leq \frac{9 d_{1}}{4 \alpha}\left\|A_{n+1}^{0}-\tilde{A}_{n}\right\|_{\mathcal{L}\left(X_{n+1}, Y\right)}\left\|e_{n}\right\|_{X_{n}} .
\end{aligned}
$$

From the definition of $A_{n+1}^{0}$ and $\tilde{A}_{n}$ we have

$$
\begin{aligned}
\left\|A_{n+1}^{0}-\tilde{A}_{n}\right\|_{\mathcal{L}\left(X_{n+1}, Y\right) \leq} & \left\|\partial_{r} \tilde{F}\left(r_{n}, k_{n+1}\right)-\partial_{r} \tilde{F}\left(r_{n}, k_{n}\right)\right\|_{\mathcal{L}\left(X_{n+1}, Y\right)} \\
& +\left\|\partial_{r} \tilde{F}\left(r_{n}, k_{n}\right)-\partial_{r} \tilde{F}\left(\tilde{r}\left(k_{n}\right), k_{n}\right)\right\|_{\mathcal{L}\left(X_{n+1}, Y\right)} \\
\leq & \Delta k d_{4}+d_{3}\left\|e_{n}\right\|_{X_{n}}
\end{aligned}
$$


Replacing this estimate into the above inequality we obtain

$$
\left\|\left(\tilde{R}_{n}-R_{n+1}^{0}\right) \tilde{F}\left(r_{n}, k_{n}\right)\right\|_{X_{n+1}} \leq \frac{9 d_{1}}{4 \alpha}\left(\Delta k d_{4}+d_{3}\left\|e_{n}\right\|_{X_{n}}\right)\left\|e_{n}\right\|_{X_{n+1}} .
$$

It follows from (4.8) that

$$
\left\|R_{n+1}^{0}\left[\tilde{F}\left(r_{n}, k_{n+1}\right)-\tilde{F}\left(r_{n}, k_{n}\right)\right]\right\|_{X_{n+1}} \leq \frac{d_{2}}{2 \sqrt{\alpha}} \Delta k .
$$

Substituting (4.21)-(4.23) into (4.20), we obtain

$$
\left\|\tilde{R}_{n} \tilde{A}_{n} e_{n}+R_{n+1}^{0} \tilde{F}\left(r_{n}, k_{n+1}\right)\right\|_{X_{n+1}} \leq \frac{d_{2}}{2 \sqrt{\alpha}} \Delta k+\left(\frac{9 d_{1} d_{3}}{4 \alpha}+\frac{d_{3}}{4 \sqrt{\alpha}}\right)\left\|e_{n}\right\|_{X_{n}}^{2} .
$$

From (4.17)-(4.19) and (4.24) we have

$$
\begin{aligned}
\left\|\mathrm{e}_{n+1}^{1}\right\|_{X_{n+1}} \leq & \Delta k\left(d_{0}+\frac{d_{2}}{2 \sqrt{\alpha}}\right)+\frac{\alpha}{\alpha+\sigma^{2}}\left\|e_{n}\right\|_{X_{n}} \\
& +\frac{9 d_{1} d_{4}}{4 \alpha} \Delta k\left\|e_{n}\right\|_{X_{n}}+\left(\frac{9 d_{1} d_{3}}{4 \alpha}+\frac{d_{3}}{4 \sqrt{\alpha}}\right)\left\|e_{n}\right\|_{X_{n}}^{2} .
\end{aligned}
$$

Let us estimate the right hand side of (4.25). First, it follows from (4.13) that

$$
\frac{\alpha}{\alpha+\sigma^{2}} \leq \frac{\epsilon}{3}
$$

Next, if $\left\|e_{n}\right\|_{X_{n}} \leq d_{0} c_{0} \alpha$, from (4.15) we have

$$
\left(\frac{9 d_{1} d_{3}}{4 \alpha}+\frac{d_{3}}{4 \sqrt{\alpha}}\right)\left\|e_{n}\right\|_{X_{n}}=\frac{\epsilon}{3 c_{0} \alpha}\left\|e_{n}\right\|_{X_{n}} \leq \frac{d_{0} \epsilon}{3} .
$$

For the chosen $\alpha$, we can also choose a number $N_{0}=N_{0}(\alpha)$ such that for all $N \geq N_{0}$, we have

$$
\frac{9 d_{1} d_{4}}{4 \alpha} \Delta k \leq \frac{\epsilon}{3}
$$

and

$$
\Delta k\left(1+\frac{d_{2}}{2 d_{0} \sqrt{\alpha}}\right) \leq\left[1-\frac{\epsilon}{3}\left(2+d_{0}\right)\right] c_{0} \alpha .
$$

Note that the right hand side is positive. It follows from (4.25)-(4.29) that

$$
\left\|\mathrm{e}_{n+1}^{1}\right\|_{X_{n+1}} \leq\left[1-\frac{\epsilon}{3}\left(2+d_{0}\right)\right] d_{0} c_{0} \alpha+\frac{\epsilon}{3}\left(2+d_{0}\right)\left\|e_{n}\right\|_{X_{n}} \leq d_{0} c_{0} \alpha .
$$

Next, we estimate $\mathrm{e}_{n+1}^{j+1}$ for $j=1, \ldots, J-1$. We rewrite them in the form

$$
\begin{aligned}
\mathrm{e}_{n+1}^{j+1} & =\tilde{r}\left(k_{n+1}\right)-r_{n+1}^{j}+R_{n+1}^{j} \tilde{F}\left(r_{n+1}^{j}, k_{n+1}\right) \\
& =\mathrm{e}_{n+1}^{j}-\tilde{R}_{n+1}^{j} \tilde{A}_{n+1} \mathrm{e}_{n+1}^{j}+\tilde{R}_{n+1}^{j} \tilde{A}_{n+1} \mathrm{e}_{n+1}^{j}+R_{n+1}^{j} \tilde{F}\left(r_{n+1}^{j}, k_{n+1}\right) .
\end{aligned}
$$

By the same arguments as above, we obtain

$$
\left\|\mathrm{e}_{n+1}^{j+1}\right\|_{X_{n+1}} \leq \frac{\alpha}{\alpha+\sigma^{2}}\left\|\mathrm{e}_{n+1}^{j}\right\|_{X_{n+1}}+\left(\frac{9 d_{1} d_{3}}{4 \alpha}+\frac{d_{3}}{4 \sqrt{\alpha}}\right)\left\|\mathrm{e}_{n+1}^{j}\right\|_{X_{n+1}}^{2} .
$$


Hence, under the same conditions (4.26) and (4.27)

$$
\left\|\mathrm{e}_{n+1}^{j+1}\right\|_{X_{n+1}} \leq \frac{\epsilon}{3}\left(1+d_{0}\right)\left\|\mathrm{e}_{n+1}^{j}\right\|_{X_{n+1}}<\left\|\mathrm{e}_{n+1}^{j}\right\|_{X_{n+1}}, j=1, \ldots, J-1
$$

Therefore, if $\left\|e_{0}\right\|_{X_{0}} \leq d_{0} c_{0} \alpha$, we can prove by recurrence that $\left\|\mathrm{e}_{n}^{j}\right\|_{X_{n}} \leq d_{0} c_{0} \alpha$ for $j=1, \ldots, J$ and $n=1, \ldots, N$. From this it is clear that

$$
r_{n}^{j}(t) \geq \tilde{r}\left(k_{n}, t\right)-\mathrm{e}_{n}^{j}(t) \geq \tilde{c}-d_{0} c_{0} \alpha>0 \text { for all } j \text { and } n .
$$

Hence, $r_{n}(t)>0$ for all $n$. Moreover,

$$
\left\|e_{n+1}\right\|_{X_{n+1}} \leq\left(\frac{\epsilon\left(1+d_{0}\right)}{3}\right)^{J-1}\left[\Delta k\left(d_{0}+\frac{d_{2}}{2 \sqrt{\alpha}}\right)+\frac{\epsilon\left(2+d_{0}\right)}{3}\left\|e_{n}\right\|_{X_{n}}\right], \forall n=0, \ldots, N-1 .
$$

Consequently,

$$
\begin{aligned}
\left\|e_{N}\right\|_{X_{N}} \leq & \left(\frac{\epsilon\left(1+d_{0}\right)}{3}\right)^{J-1} \Delta k\left(d_{0}+\frac{d_{2}}{2 \sqrt{\alpha}}\right) \frac{1}{1-\left(\frac{\epsilon\left(1+d_{0}\right)}{3}\right)^{J-1} \frac{\epsilon\left(2+d_{0}\right)}{3}} \\
& +\left(\frac{\epsilon\left(1+d_{0}\right)}{3}\right)^{(J-1) N}\left(\frac{\epsilon\left(2+d_{0}\right)}{3}\right)^{N}\left\|e_{0}\right\|_{X_{0}} \\
\leq & \left(\frac{\epsilon\left(1+d_{0}\right)}{3}\right)^{J-1} \frac{\Delta k}{\sqrt{\alpha}}\left\{\left(d_{0} \frac{\sqrt{\epsilon} \sigma}{\sqrt{3-\epsilon}}+\frac{d_{2}}{2}\right) \frac{1}{1-\left(\frac{\epsilon\left(1+d_{0}\right)}{3}\right)^{J-1} \frac{\epsilon\left(2+d_{0}\right)}{3}}\right. \\
& \left.+\left(\frac{\epsilon\left(1+d_{0}\right)}{3}\right)^{(J-1)(N-1)} \frac{N\left(\frac{\epsilon\left(2+d_{0}\right)}{3}\right)^{N}}{k_{h}-k_{l}} d_{0} c_{0}\left(\frac{\sqrt{\epsilon} \sigma}{\sqrt{3-\epsilon}}\right)^{3}\right\} .
\end{aligned}
$$

From (4.15) we can see that $c_{0}$ is bounded from above by

$$
c_{0} \leq \frac{4 \epsilon}{27 d_{1} d_{3}} .
$$

Moreover, for a fixed frequency interval $\left[k_{l}, k_{h}\right], N \epsilon^{N}$ is bounded in terms of $N$. Therefore, there exists a constant $C^{*}>0$ independent of $N$ and $\alpha$ such that

$$
\left(d_{0} \frac{\sqrt{\epsilon} \sigma}{\sqrt{3-\epsilon}}+\frac{d_{2}}{2}\right) \frac{1}{1-\left(\frac{\epsilon\left(1+d_{0}\right)}{3}\right)^{J-1} \frac{\epsilon\left(2+d_{0}\right)}{3}}+\left(\frac{\epsilon\left(1+d_{0}\right)}{3}\right)^{(J-1)(N-1)} \frac{N\left(\frac{\epsilon\left(2+d_{0}\right)}{3}\right)^{N}}{k_{h}-k_{l}} d_{0} c_{0}\left(\frac{\sqrt{\epsilon} \sigma}{\sqrt{3-\epsilon}}\right)^{3} \leq C^{*} .
$$

On the other hand, it follows from (4.28) that $\sqrt{\frac{\Delta k}{\alpha}} \leq \sqrt{\frac{4 \epsilon}{27 d_{1} d_{4}}}$. Replacing these inequalities into (4.32) we obtain (4.16) with $C_{1}=C^{*} \sqrt{\frac{4 \epsilon}{27 d_{1} d_{4}}}$.

In the case of noisy data, we have the following result.

Theorem 4.6. Assume that the radial functions $\tilde{r}\left(k_{n}\right)$ and the subspaces $X_{n}, n=0, \ldots, N$, are as in Theorem 4.5. Let $r_{n}, n=0, \ldots, N$, be given by Algorithm 4.1. For fixed positive real numbers $\epsilon$, $\xi$, 
$0<\epsilon<3 /\left(2+d_{0}\right), 0<\xi<1$, and for the parameters $\alpha$ and $c_{0}$ satisfying (4.13) and (4.15) respectively, we define the positive parameter $\delta_{0}$ by

$$
\delta_{0}:=\frac{2 \xi}{\tau}\left[1-\frac{\epsilon\left(2+d_{0}\right)}{3}\right] d_{0} c_{0} \alpha^{3 / 2} .
$$

Then there exists an integer $N_{0}$ independent of $\delta$ such that if (4.14) is satisfied, we have $r_{n}(t)>0 \forall t \in[0,2 \pi]$ and the following error estimate holds true

$$
\left\|\tilde{r}\left(k_{h}\right)-r_{N}\right\|_{X_{N}} \leq C_{2} \delta^{2 / 3}+\left(\frac{\epsilon\left(1+d_{0}\right)}{3}\right)^{J-1} C_{1} \sqrt{\Delta k}, \forall N \geq N_{0}
$$

for every $\delta \leq \delta_{0}$, where $C_{1}$ is as in Theorem 4.5 and $C_{2}$ is a constant independent of $\delta, \alpha$ and $N$.

Proof. Using (4.6) we can rewrite the error as

$$
\mathrm{e}_{n+1}^{j+1}=\tilde{r}\left(k_{n+1}\right)-r_{n+1}^{j}+R_{n+1}^{j} \tilde{F}\left(r_{n+1}^{j}, k_{n+1}\right)+R_{n+1}^{j} f^{\delta}\left(\tilde{r}\left(k_{n+1}\right), k_{n+1}\right)
$$

for $j=0, \ldots, J-1$. It follows from Lemma 4.4 that

$$
\left\|R_{n+1}^{j} f^{\delta}\left(\tilde{r}\left(k_{n+1}\right), k_{n+1}\right)\right\|_{X_{n+1}} \leq \frac{\tau \delta}{2 \sqrt{\alpha}} .
$$

Using the estimates (4.25) and (4.31) for the noiseless case, from (4.37)-(4.38) we have

$$
\begin{aligned}
\left\|\mathrm{e}_{n+1}^{1}\right\|_{X_{n+1}} \leq & \Delta k\left(d_{0}+\frac{d_{2}}{2 \sqrt{\alpha}}\right)+\frac{\tau \delta}{2 \sqrt{\alpha}}+\frac{\alpha}{\alpha+\sigma^{2}}\left\|e_{n}\right\|_{X_{n}} \\
& +\frac{9 d_{1} d_{4}}{4 \alpha} \Delta k\left\|e_{n}\right\|_{X_{n}}+\left(\frac{9 d_{1} d_{3}}{4 \alpha}+\frac{d_{3}}{4 \sqrt{\alpha}}\right)\left\|e_{n}\right\|_{X_{n}}^{2} .
\end{aligned}
$$

And for $j=1, \ldots, J-1$, we obtain

$$
\left\|\mathrm{e}_{n+1}^{j+1}\right\|_{X_{n+1}} \leq \frac{\tau \delta}{2 \sqrt{\alpha}}+\frac{\alpha}{\alpha+\sigma^{2}}\left\|\mathrm{e}_{n+1}^{j}\right\|_{X_{n+1}}+\left(\frac{9 d_{1} d_{3}}{4 \alpha}+\frac{d_{3}}{4 \sqrt{\alpha}}\right)\left\|\mathrm{e}_{n+1}^{j}\right\|_{X_{n+1}}^{2} .
$$

For $\delta \leq \delta_{0}$, we have from (4.13) and (4.35) that

$$
\left(\frac{\tau \delta}{2 \xi\left[1-\frac{\epsilon\left(2+d_{0}\right)}{3}\right] d_{0} c_{0}}\right)^{2 / 3} \leq \alpha \leq \frac{\epsilon}{3-\epsilon} \sigma^{2} .
$$

Or, equivalently, $\alpha$ satisfies (4.26) and the following inequality

$$
\frac{\tau \delta}{2 \sqrt{\alpha}} \leq \xi\left[1-\frac{\epsilon\left(2+d_{0}\right)}{3}\right] d_{0} c_{0} \alpha
$$

On the other hand, there exists $N_{0}$ such that condition (4.28) is satisfied for all $N>N_{0}$ and

$$
\Delta k\left(d_{0}+\frac{d_{2}}{2 \sqrt{\alpha}}\right) \leq(1-\xi)\left[1-\frac{\epsilon\left(2+d_{0}\right)}{3}\right] d_{0} c_{0} \alpha,
$$

Now using the same arguments as in the proof of Theorem 4.5, we can show that $\left\|\mathrm{e}_{n}^{j}\right\|_{X_{n}} \leq d_{0} c_{0} \alpha$ for all $j=0, \ldots, J ; n=1, \ldots, N$, if (4.14) is satisfied. This implies the positivity of $r_{n}$ as in Theorem 4.5. Moreover,

$$
\begin{aligned}
& \left\|\mathrm{e}_{n+1}^{1}\right\|_{X_{n+1}} \leq \Delta k\left(d_{0}+\frac{d_{2}}{2 \sqrt{\alpha}}\right)+\frac{\tau \delta}{2 \sqrt{\alpha}}+\frac{\epsilon\left(2+d_{0}\right)}{3}\left\|e_{n}\right\|_{X_{n}}, \\
& \left\|\mathrm{e}_{n+1}^{j+1}\right\|_{X_{n+1}} \leq \frac{\tau \delta}{2 \sqrt{\alpha}}+\frac{\epsilon\left(1+d_{0}\right)}{3}\left\|\mathrm{e}_{n+1}^{j}\right\|_{X_{n+1}}, j=1, \ldots, J-1 .
\end{aligned}
$$


Consequently, for $n=0, \ldots, N-1$, we have

$$
\left\|e_{n+1}\right\|_{X_{n+1}} \leq \frac{\tau \delta}{2 \sqrt{\alpha}} \frac{1}{1-\frac{\epsilon\left(1+d_{0}\right)}{3}}+\left(\frac{\epsilon\left(1+d_{0}\right)}{3}\right)^{J-1}\left[\Delta k\left(d_{0}+\frac{d_{2}}{2 \sqrt{\alpha}}\right)+\frac{\epsilon\left(2+d_{0}\right)}{3}\left\|e_{n}\right\|_{X_{n}}\right] .
$$

Hence,

$$
\begin{aligned}
\left\|e_{N}\right\|_{X_{N}} \leq & \frac{\tau \delta}{2 \sqrt{\alpha}\left[1-\frac{\epsilon\left(1+d_{0}\right)}{3}\right]\left[1-\left(\frac{\epsilon\left(1+d_{0}\right)}{3}\right)^{J-1} \frac{\epsilon\left(2+d_{0}\right)}{3}\right]} \\
& +\left(\frac{\epsilon\left(1+d_{0}\right)}{3}\right)^{J-1} C_{1} \sqrt{\Delta k}
\end{aligned}
$$

Here the constant $C$ is the same as in Theorem 4.5. Finally, taking into account the condition (4.41) we obtain (4.36) with the constant $C_{2}$ given by

$$
C_{2}=\frac{\tau^{2 / 3}\left(\xi d_{0} \frac{4 \epsilon}{27 d_{1} d_{3}}\right)^{1 / 3}}{\left[2\left(1-\frac{\epsilon\left(1+d_{0}\right)}{3}\right)\right]^{2 / 3}\left[1-\left(\frac{\epsilon\left(1+d_{0}\right)}{3}\right)^{J-1} \frac{\epsilon\left(2+d_{0}\right)}{3}\right]}
$$

The proof is complete.

Remark 4.7. To obtain the Hölder type error estimate of the form $\left\|e_{N}\right\|_{X_{n+1}}=O\left(\delta^{2 / 3}\right)$, we require that $\left(\frac{\epsilon\left(1+d_{0}\right)}{3}\right)^{J-1} \sqrt{\Delta k}=O\left(\delta^{2 / 3}\right)$. That means, if $\Delta k$ is small, we do not need to use many Newton iterations and vice versa. In other words, there is a trade-off between the frequency step $\Delta k$ and the number of Newton iterations for a given accuracy.

\subsection{Discussion on the link between the true shape and the observable shapes}

Theorems 4.5 and 4.6 show the accuracy of the reconstruction of the observable shapes. The final accuracy of the algorithm with respect to the true shape depends on the stability of the reconstruction problem under investigation and the dependency of the constants $C_{1}$ and $C_{2}$ in the error estimates of Theorems 4.5 and 4.6 on the frequency interval. Concerning the first point, at low or moderate frequencies, the stability of the inverse problem is of log type, see, e.g., [18,27]; when the final frequency $k_{h}$ is very high and the obstacle is convex, a Hölder type stability estimate was proved in [28] for the part of the boundary illuminated by the incident wave. Concerning the second point, a rigorous answer is still open. Below we give a heuristic, non-rigorous explanation about which factors could affect the error estimates when $k_{h}$ increases. For simplicity, we assume that the lowest frequency is fixed and the same frequency step is used in all frequency intervals.

First of all, we know that the higher the frequency, the better the stability of the reconstruction problem. Therefore the observable shapes should become closer and closer. As a result, the constant $d_{0}$ in Assumption 2 should not increase when $k_{h}$ is increased. Second, we can see from (4.8) that $d_{1}, d_{2}$ and $d_{3}$ are non-decreasing. Moreover, since $c_{0}$ can be bounded from above by a constant which is not increased when $k_{h}$ increases, see (4.33), the constant $C_{2}$ is non-increasing.

Concerning the constant $C_{1}$, from (4.34) it follows that the second term is non-increasing. Indeed, for a given frequency step $\Delta k$, we have

$$
\frac{N\left(\frac{\epsilon\left(2+d_{0}\right)}{3}\right)^{N}}{k_{h}-k_{l}}=\frac{\left(\frac{\epsilon\left(2+d_{0}\right)}{3}\right)^{N}}{\Delta k} .
$$

That means, it is non-increasing when $k_{h}$ increases if the frequency step is kept fixed. The other factors of the second term of (4.34) are clearly non-increasing. Hence, the only factor which could cause the constant $C_{1}$ to increase is $d_{2}$ in the first term of (4.34). 
The question on how this factor $d_{2}$ depends on the frequency is still open to us. Note however that, based on integral equation methods, precisely the explicit dependence of the norms of the corresponding boundary integral operators in terms of the frequencies, see $[5,23]$ for instance, we infer that $d_{2}$ increases as $k_{h}$ increases, but at a moderate rate, i.e., polynomially. Then we can eliminate its effect on the constant $C_{1}$ by increasing the number of Newton steps at each frequency. We will investigate this question in a future work.

\section{Multi-Level Newton method}

In this Section, we discuss how to obtain the comparable error estimates as in the previous Section but with a less restrictive condition than (4.14) concerning the reconstruction at the lowest frequency. For this purpose, we use a multi-level Newton method which is described hereafter.

We recall that the error estimate (4.36) was obtained under the conditions (4.26), (4.28), (4.41) and (4.42). In this Section, we choose $\xi=1 / 2$ for simplicity. To make the analysis easier to follow, we rewrite the above conditions here

$$
\begin{aligned}
\alpha & \leq \frac{\epsilon \sigma^{2}}{3-\epsilon}, \\
\frac{9 d_{1} d_{4}}{4 \alpha} \Delta k & \leq \frac{\epsilon}{3}, \\
\Delta k\left(d_{0}+\frac{d_{2}}{2 \sqrt{\alpha}}\right) & \leq \frac{1}{2}\left[1-\frac{\epsilon\left(2+d_{0}\right)}{3}\right] d_{0} c_{0} \alpha, \\
\frac{\tau \delta}{2 \sqrt{\alpha}} & \leq \frac{1}{2}\left[1-\frac{\epsilon\left(2+d_{0}\right)}{3}\right] d_{0} c_{0} \alpha,
\end{aligned}
$$

with the constant $c_{0}$ being given by (4.15) which depends on $\alpha$. Therefore, in the following, we denote by $c_{0}(\alpha)$ to indicate this dependence. We reserve the notations $c_{0}$ and $\alpha$ for the constants in the previous Section, i.e., these constants associate with the full frequency set. So Theorem 4.6 says that if the conditions (5.1)-(5.4) are satisfied, and if the solution $r_{0}$ at the lowest wavenumber $k_{0}$ satisfies $(4.14)$, i.e.,

$$
\left\|\tilde{r}\left(k_{l}\right)-r_{0}\right\|_{X} \leq d_{0} c_{0} \alpha<\tilde{c},
$$

then the final error estimate (4.36) holds true.

We remark that the regularization parameter $\alpha$ depends on the smallest singular value $\sigma$ of the domain derivative $\tilde{A}_{n}, n=0,1, \ldots, N$. Clearly, the more frequencies used, the smaller this singular value $\sigma$ is. Therefore, by subdividing the original interval of frequencies into sub-intervals and choosing this regularization parameter depending on the smallest singular value in different frequency sub-intervals, we may not need to choose a small regularization parameter (in other words, with a less restrictive condition on the initial guess) at the first sub-interval but still obtain a comparable error estimate as (4.36).

To make the following analysis consistent with the previous Section, we still consider the set of frequencies $k_{0}=k_{l}, \ldots, k_{N}=k_{h}$ with step size $\Delta k$ as in Section 4 . Suppose that the original frequency interval $\left\{k_{0}, \ldots, k_{N}\right\}$ is divided into $M$ sub-intervals from low to high frequencies. These sub-intervals do not need to have the same number of frequencies. We denote by $\tilde{\sigma}_{m}$ the smallest singular value in the $m$ th sub-interval. That is,

$$
\tilde{\sigma}_{m}=\min \left\{\sigma_{n}, k_{n} \text { belongs to the } m \text {-th sub-interval }\right\} \text {. }
$$

Here $\sigma_{n}$ the smallest singular value of $\tilde{A}_{n}$ as in Section 4. Moreover, we choose the sequence of parameters $\hat{\sigma}_{m}, m=1, \ldots, M$, as follows:

$$
\hat{\sigma}_{1}=\tilde{\sigma}_{1}, \quad \hat{\sigma}_{m+1}=\min \left\{\hat{\sigma}_{m}, \tilde{\sigma}_{m+1}\right\}, m=1, \ldots, M-1 .
$$

by this choice of the parameters $\hat{\sigma}_{m}$, it is clear that

$$
\hat{\sigma}_{1} \geq \hat{\sigma}_{2} \geq \cdots \geq \hat{\sigma}_{M} \geq \sigma .
$$


Associated with these sub-intervals, we choose the set of regularization parameters $\alpha_{m}, m=1, \ldots, M$ such that (5.1) is satisfied in each sub-intervals, where $\sigma$ is replaced by the corresponding parameter $\hat{\sigma}_{m}$. That is,

$$
\alpha_{m} \leq \frac{\epsilon \hat{\sigma}_{m}^{2}}{3-\epsilon}, m=1, \ldots, M
$$

Moreover, $\alpha_{m}$ are also chosen such that

$$
\alpha_{1} \geq \alpha_{2} \geq \cdots \geq \alpha_{M} \geq \alpha .
$$

The multi-level Newton algorithm can be summarized as follows.

\section{Algorithm 5.1.}

- Given measured data $u_{m}^{\infty, \delta}(\cdot, k)$ for $k=k_{0}, \ldots, k_{N}$, and the partition of this frequency interval into $M$ sub-intervals.

- Step 1: Choose a subspace $X_{0}$ and find an approximation $r_{0} \in X_{0}$ at wavenumber $k_{0}$.

- Step 2: For $m=1, \ldots, M$

- Choose $\alpha_{m}$ satisfying (5.7) and (5.8).

- Use Algorithm 4.1 to find an approximation in the $m$-th frequency sub-interval.

Let us show a similar convergence result as in Theorem 4.6 for this algorithm. From (4.15) and (5.8) it can be proved using elementary analysis that

$$
c_{0}\left(\alpha_{1}\right) \alpha_{1} \geq c_{0}\left(\alpha_{2}\right) \alpha_{2} \geq \cdots \geq c_{0}\left(\alpha_{M}\right) \alpha_{M} \geq c_{0} \alpha .
$$

We recall that $c_{0}$ and $\alpha$ are associated with the whole frequency interval $\left\{k_{0}, \ldots, k_{N}\right\}$. It also follows from (5.8) and (5.9) that Inequalities (5.2)-(5.4) still hold for the same frequency step $\Delta k$ and noise level as in Theorems 4.5 and 4.6 when $\alpha$ is replaced by $\alpha_{m}$ and $c_{0}$ by $c_{0}\left(\alpha_{m}\right)$. That means, all the conditions of these theorems are satisfied for each sub-interval.

Now we replace the condition (4.14) in Theorems 4.5 and 4.6 by the following one for the first sub-interval:

$$
\left\|\tilde{r}\left(k_{l}\right)-r_{0}\right\|_{X} \leq d_{0} c_{0}\left(\sigma_{1}\right) \alpha_{1}<\tilde{c} .
$$

Hence, from Theorem 4.6 (see (4.45)) we obtain the following error estimate in the first sub-interval

$$
\begin{aligned}
& \left\|\tilde{r}\left(k_{N_{1}}\right)-r_{N_{1}}\right\|_{X} \leq \frac{\tau \delta}{2 \sqrt{\alpha_{1}}\left[1-\frac{\epsilon\left(1+d_{0}\right)}{3}\right]\left[1-\left(\frac{\epsilon\left(1+d_{0}\right)}{3}\right)^{J-1} \frac{\epsilon\left(2+d_{0}\right)}{3}\right]} \\
& +\tilde{C}_{1}\left(\frac{\epsilon\left(1+d_{0}\right)}{3}\right)^{J-1} \sqrt{\Delta k} . \\
& \leq \frac{\tau \delta}{2 \sqrt{\alpha}\left[1-\frac{\epsilon\left(1+d_{0}\right)}{3}\right]\left[1-\left(\frac{\epsilon\left(1+d_{0}\right)}{3}\right)^{J-1} \frac{\epsilon\left(2+d_{0}\right)}{3}\right]} \\
& +\tilde{C}_{1}\left(\frac{\epsilon\left(1+d_{0}\right)}{3}\right)^{J-1} \sqrt{\Delta k} .
\end{aligned}
$$

for a constant $\tilde{C}_{1}$. This constant can be chosen fixed for all frequency sub-intervals and independent of $\delta$. Here $k_{N_{1}}$ is the maximum frequency of the first sub-interval. It follows from (5.3) and (5.11) that

$$
\left\|\tilde{r}\left(k_{N_{1}}\right)-r_{N_{1}}\right\|_{X} \leq \frac{d_{0} c_{0} \alpha}{2\left[1-\left(\frac{\epsilon\left(1+d_{0}\right)}{3}\right)^{J-1} \frac{\epsilon\left(2+d_{0}\right)}{3}\right]}+\tilde{C}_{1}\left(\frac{\epsilon\left(1+d_{0}\right)}{3}\right)^{J-1} \sqrt{\Delta k}
$$


In the second sub-interval, we use the final approximation $r_{N_{1}}$ of the first sub-interval as the initial guess, i.e., it plays the same role as $r_{0}$ in Section 4 . For the given frequency step $\Delta k$, we can choose the number of Newton iterations $J$ large enough so that the following inequality holds true

$$
\left\|\tilde{r}\left(k_{N_{1}}\right)-r_{N_{1}}\right\|_{X} \leq d_{0} c_{0} \alpha_{2} .
$$

This process can be continued until the last sub-interval. In the last sub-interval, we obtain a similar error estimate as (4.36). We summarize the above analysis in the following theorem.

Theorem 5.2. Suppose that the frequency set $\left\{k_{0}, \ldots, k_{N}\right\}$ is subdivided into $M$ sub-intervals. Denote by $N_{m}$ the number of frequencies in the mth sub-interval. Moreover, let $\epsilon$ be a positive real number satisfying $0<$ $\epsilon<3 /\left(2+d_{0}\right)$, and $\alpha_{m}, m=1, \ldots, M$, be the regularization parameters satisfying (5.7) and (5.8). We also suppose that the frequency step is small enough so that the conditions (5.2) and (5.3) are fulfilled for $\alpha=$ $\min \left\{\alpha_{m}, m=1, \ldots, M\right\}$ and $c_{0}=c_{0}(\alpha)$ given by (4.15). Then there exists an integer $J$ large enough such that if the reconstruction at the lowest frequency satisfies (5.10), we have $r_{n}(t)>0 \forall t \in[0,2 \pi]$ and the following error estimate holds true

$$
\left\|\tilde{r}\left(k_{h}\right)-r_{N}\right\|_{X} \leq C_{2} \delta^{2 / 3}+\left(\frac{\epsilon\left(1+d_{0}\right)}{3}\right)^{J-1} \tilde{C}_{1} \sqrt{\Delta k},
$$

for every $\delta \leq \delta_{0}$, where $C_{2}$ is as in Theorem 4.6 and $\tilde{C}_{1}$ is a constant independent of $\delta, \alpha_{m}$ and $N$. Here $\delta_{0}$ is defined as in (4.35).

Remark 5.3. Theorem 5.2 indicates that we still obtain the same error estimate as in Theorem 4.6 with $C_{1}$ being replaced by $\tilde{C}_{1}$. That means, by using the multi-level algorithm, we can obtain basically the same error estimate as in Theorem 4.6 with the first guess $r_{0}$ satisfying the condition (5.10) which is, in general, weaker than (4.14) due to (5.9). This issue is related to estimating the lower bounds of the singular values $\sigma_{1}$. Actually, at each level $m, m=1, \ldots, M$, we take the regularization parameter $\alpha$ satisfying a similar estimate, i.e., (5.7). In a forthcoming work, we will investigate the lower bound of $\sigma_{m}$ in terms of the wavenumber $k$ and the dimension of the corresponding subspace $X_{n+1}$. With such estimates at hand, the regularization parameter $\alpha_{m}$ can be chosen depending on the known quantities $k$ and $n$. Let us finally make some comments on the condition (5.4) on the noise level. As the frequency becomes high, $\alpha$ becomes small and so for the noise level. However this is quite natural since at high frequencies we expect to reconstruct small details and this makes sense only if the measurements at hand are not so noisy.

\section{Numerical Results}

In this Section, we show some numerical results to demonstrate the performance of the proposed algorithms in the previous Sections. In particular, we analyze the effect of different parameters such as the choice of the frequencies, the choice of the subspaces, and the number of Newton iterations on the reconstruction accuracy.

To the following numerical examples, we considered flower-shaped obstacles defined by the equation

$$
\left\{x(t)=x^{0}+c_{1}\left(1+c_{2} \cos c_{3} t\right)(\cos t, \sin t), t \in[0,2 \pi)\right\}
$$

with positive constants $c_{1}, c_{2}$ and $c_{3}$. The first parameter determines the area of the obstacle, the second one relates to the curvature and the last one determines the number of petals of the "flower". The obstacles are centered at $x^{0}$. Two obstacles were considered which correspond to two sets of parameters: $x^{0}=(4,0), c_{1}=2$, $c_{2}=0.3$ and $c_{3}=4$ (obstacle 1 ), and $x^{0}=(3,0), c_{1}=2, c_{2}=0.2$ and $c_{3}=9$ (obstacle 2).

The measured far field patterns $u_{m}^{\infty}\left(\cdot, k_{j}, r\right), j=0, \ldots, N$, used in these tests were simulated as the solution of the forward problem (1.1)-(1.3) which was solved by the integral equation method [10]. The same method was also used to calculate the domain derivative of the far field operator. We used $N_{a}=32$ observation directions 


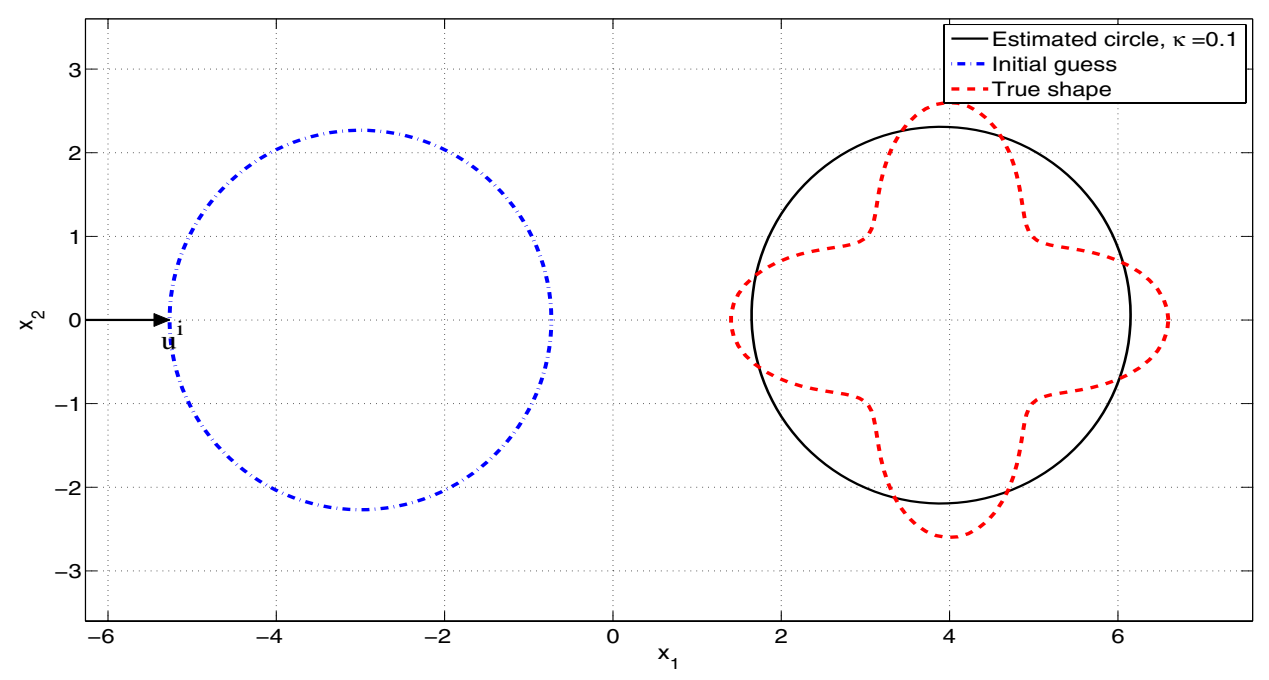

FiguRE 1. Reconstruction of an approximating circle of obstacle 1 at the lowest frequency. The initial guess for the center was chosen at $(-3,0)$. The radius of the dashed shape (the left circle) was obtained by the method of Section 3 without using a first guess.

uniformly distributed on the unit circle, $\hat{x}_{n}=(n-1) \pi / 32, n=1, \ldots, 32$. Additive random noise of $5 \%$ was added to the computed far field patterns.

Our numerical tests in [28] have indicated that although the regularization parameter $\alpha$ must satisfy conditions (4.26) and (4.41) in the theoretical analysis, numerical performance seemed to be more optimistic. In our tests, this parameter could be chosen in a wide range, say, from $10^{-6}$ to $10^{-1}$ which still provided good reconstruction results. Therefore, in the following examples, the regularization parameter $\alpha$ was chosen to be $10^{-2}$. We fixed the incident angle at $\theta=0$, i.e., $d=(1,0)$.

We first demonstrate the reconstruction at the lowest frequency using the method described in Section 3 . We note that even though the center $x^{0}$ is assumed to be fixed in the theoretical analysis, in our numerical tests we also reconstructed this point from the measured data at the lowest wavenumber $k_{0}$. The reconstruction of $x^{0}$ was done as follows. We approximated the unknown obstacle by a circle and estimated its radius $r_{0}$ using the method of Section 3. Then we measured far field pattern was approximated by

$$
u_{m}^{\infty, \delta}\left(\hat{x}_{n}, k_{0}\right) \approx \mathrm{e}^{i k_{0} x^{0} \cdot\left(d-\hat{x}_{n}\right)} u_{c}^{\infty}\left(\hat{x}_{n}, r_{0}, k_{0}\right), n=1, \ldots, N_{a} .
$$

where $u_{c}^{\infty}\left(\hat{x}, r_{0}, k_{0}\right)$ is the far field pattern of the circle $\mathbb{S}\left(0, r_{0}, k_{0}\right)$, see (3.3). For small enough wavenumber $k_{0}$, we have $\left|u_{c}^{\infty}\left(\hat{x}_{n}, r_{0}, k_{0}\right)\right|>C_{0}>0$ for some fixed constant $C_{0}$. We divided both sides of $(6.1)$ by $u_{c}^{\infty}\left(\hat{x}_{n}, r_{0}, k_{0}\right)$ and took the imaginary part both sides. Then, we found $x^{0}$ by minimizing the following least-squares function:

$$
\mathcal{F}_{2}(x)=\frac{1}{2} \sum_{n=1}^{N_{a}}\left[\sin \left(k_{0} x \cdot \varphi_{n}\right)-\psi_{n}\right]^{2},
$$

where $\varphi_{n}=d-\hat{x}_{n}$ and $\psi_{n}=\operatorname{Im}\left[u_{m}^{\infty, \delta}\left(\hat{x}, k_{0}\right) / u_{c}^{\infty}\left(\hat{x}, r_{0}, k_{0}\right)\right]$. Here $\operatorname{Im} Z$ is the imaginary part of a complex number $Z$. We have observed numerically that this objective function provided good and stable approximations of the center $x^{0}$ and does not require a good first guess. A theoretical investigation about the uniqueness and stability of this problem will be presented in an incoming work.

Figure 1 shows the reconstruction of the center of obstacle 1 at $k_{0}=0.1$. The first guess of the center $x^{0}$ was chosen to be $(-3,0)$, which was quite far from the true center. It is clear that the algorithm was able to find 


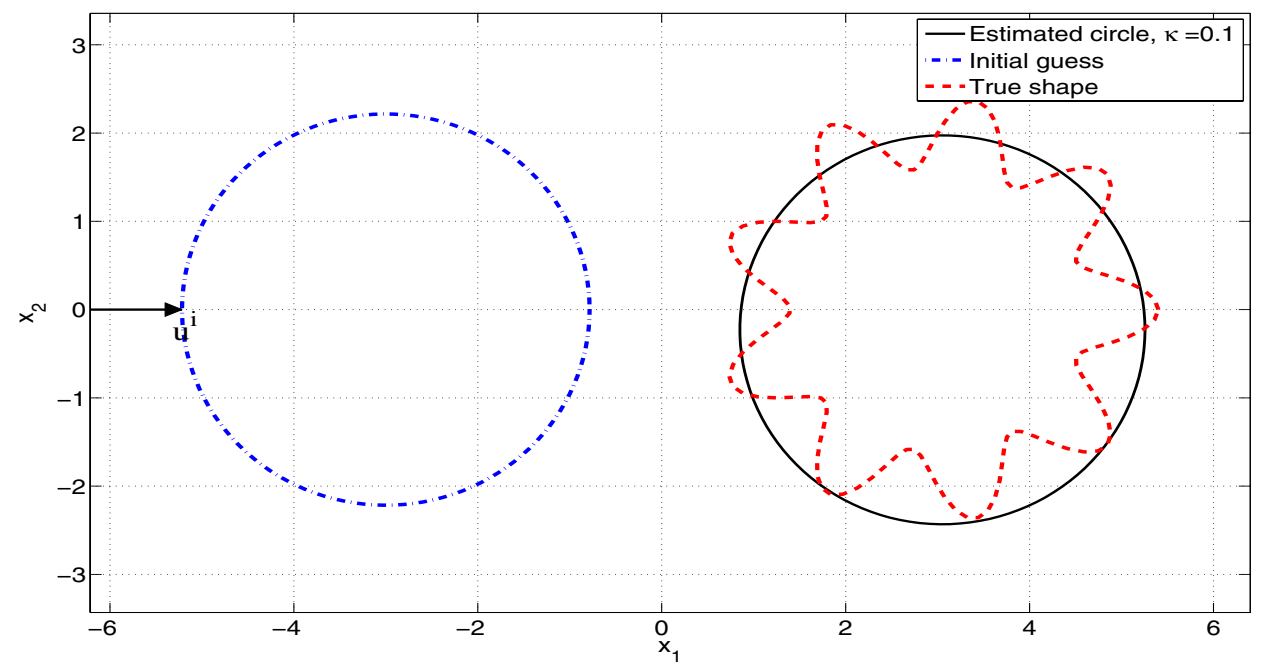

FiguRE 2. Reconstruction of an approximating circle of obstacle 2 at the lowest frequency. The initial guess for the center was chosen at $(-3,0)$.

the center of the obstacle from a first guess in a large domain. A similar result was obtained for obstacle 2 , see Figure 2 .

We should mention that when the lowest wavenumber was chosen to be $k_{0}=1$, the same initial guesss of the center did not provide good results.

The final reconstruction of the two obstacles using 20 wavenumbers from 0.1 to 8 are shown in Figure 3. Here the reconstructions at the lowest frequencies were taken as the approximating circle in Figure 1 and 2. Only one Newton iterations was taken at each frequency and the subspaces were chosen as $X_{n}=\bar{X}_{2 n}, n=0, \ldots, 19$ (see Sect. 2 for the definition of $\bar{X}_{M}$ ). As we can see, the illumiated parts of the shapes were accurately reconstructed. However, the shadowed parts were not significantly improved from the lowest frequency to the highest one.

To see the effect of the number of Newton iterations on the reconstruction accuracy, we show in Figure 4 the reconstruction of obstacle 1 using only 6 frequencies in the same interval $[0.1,8]$, starting from the same initial guess. The subspaces were also chosen the same as in the previous test shown in Figure 3. In Figure 4a only one Newton iteration was run at each frequency whereas in Figure 4(b) 10 Newton iterations were chosen. Indeed, we can observe that increasing the number of Newton iterations at each frequency helped significantly increase the accuracy.

Next, we demonstrate in Figure 5 the effect of the choice of the subspaces $X_{n}$ on the accuracy of the reconstruction. The tests were done for obstacle 2 using 10 frequencies between 0.1 and 8 . Two choices of the subspaces were taken. In Figure 5 we chose $X_{n}=\bar{X}_{2 n}, n=0, \ldots, 9$, which means that we added 2 Fourier modes from one frequency to the next one. Figure 5 (b) shows the result for $X_{n}=\bar{X}_{3 n}$. The figure indicates that if the subspaces were chosen too small, the reconstruction accuracy may be deteriorated.

Figure 6 shows that the reconstruction was still accurate for a larger set of subspaces $X_{n}=\bar{X}_{5 n}$. However, if the subspaces were chosen to be too large, the result might also be not good, as illustrated in Figure 6 where the same subspace $X_{n}=\bar{X}_{25}$ was chosen at all frequencies, while the other parameters were chosen the same as in Figure 5. Comparing these two figures we can see the effect of the choice of the subspaces on the reconstruction accuracy.

Finally, to see the performance of the multi-level method of Section 5, we show in Figure 7 the reconstruction of the two obstacles. In this test, the reconstruction $r_{0}$ at the lowest frequency was obtained at the lowest frequency of $k_{0}=0.5$. By doing so, we expected that this should not be as good as in the previous tests. Here 


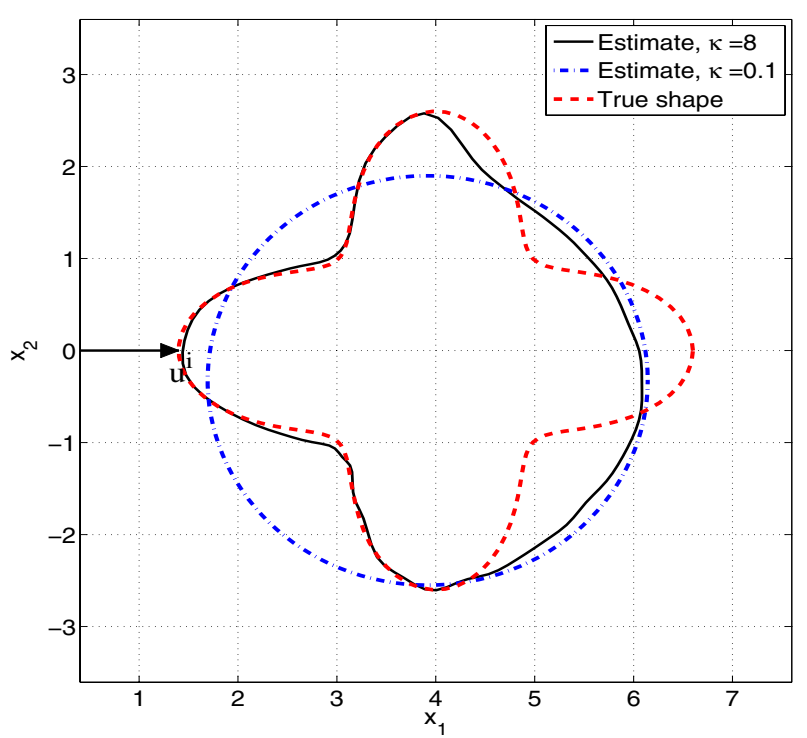

(a)

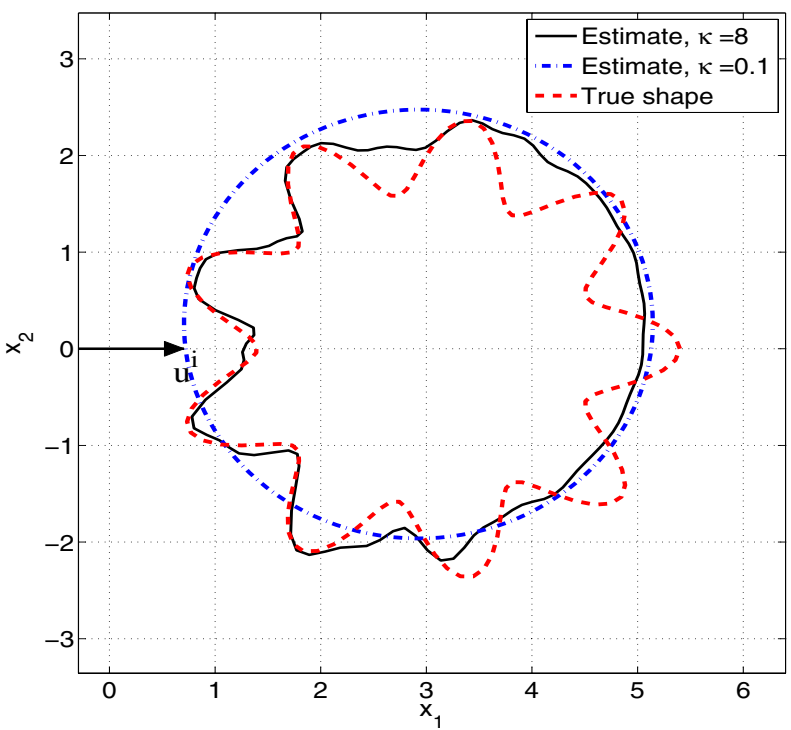

(b)

FiguRE 3. Reconstruction of obstacle 1 (a) and obstacle 2 (b) using 20 wavenumbers from 0.1 to 8 and only 1 Newton iteration at each frequency.

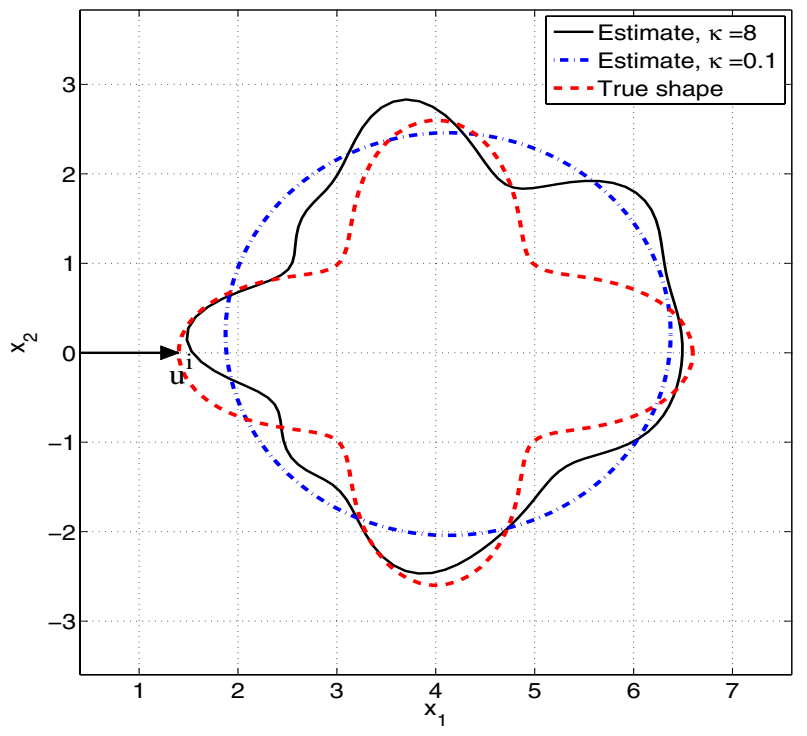

(a)

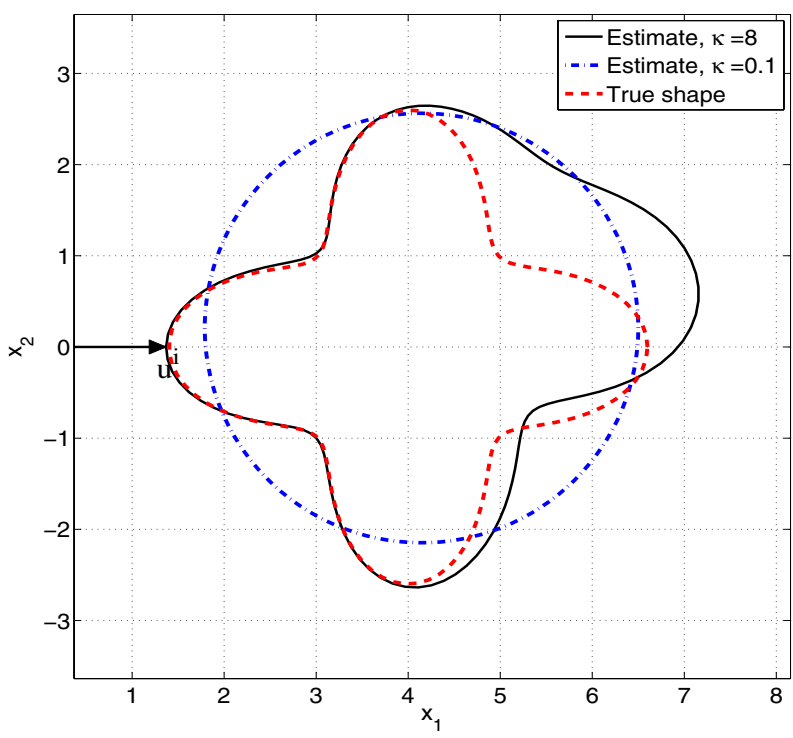

(b)

FiguRE 4. Reconstruction of obstacle 1 using 6 wavenumbers from 0.1 to 8: (a) 1 Newton iteration at each frequency, (b) 10 Newton iterations at each frequency. 


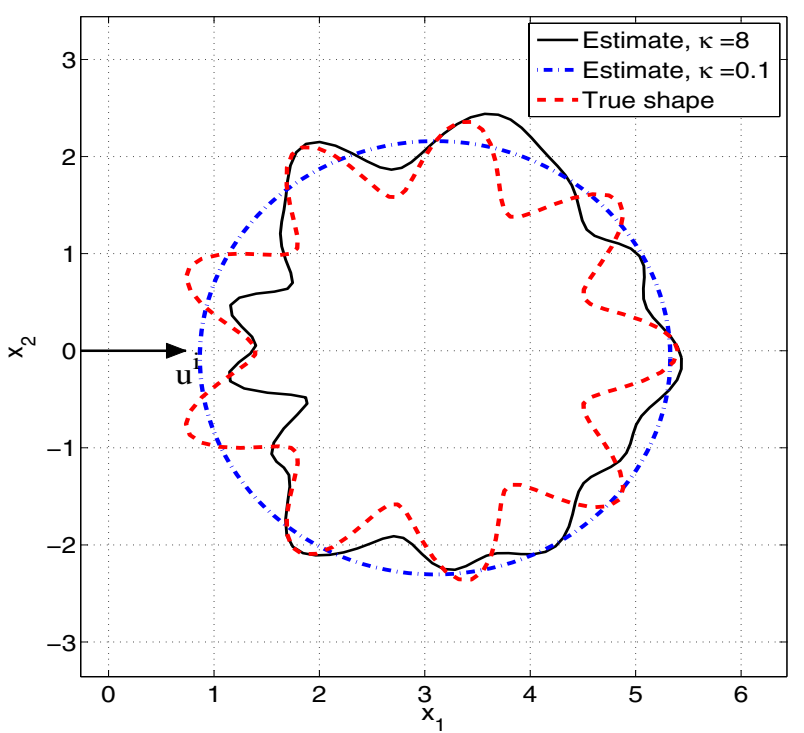

(a)

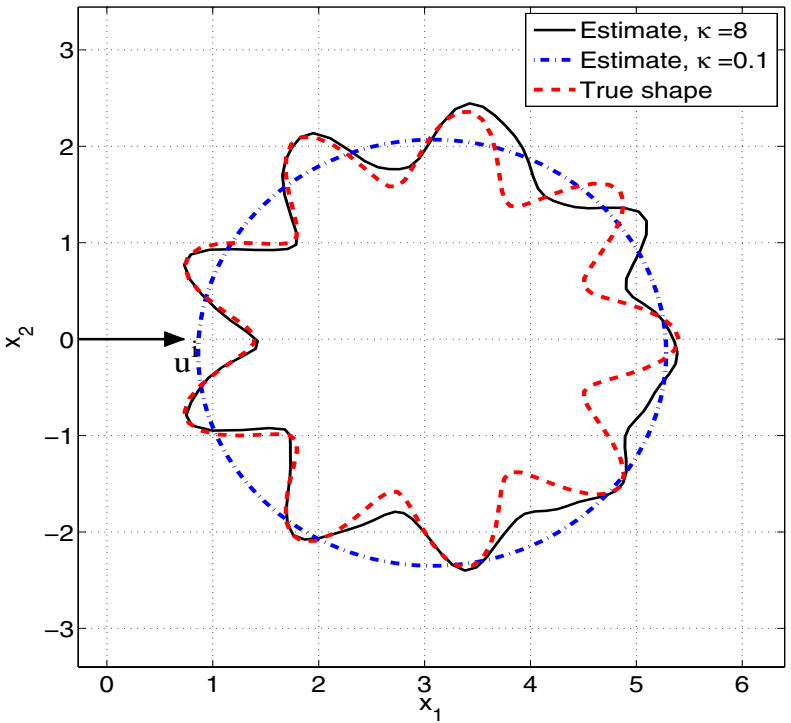

(b)

FiguRE 5. Reconstruction of obstacle 2 using 10 wavenumbers from 0.1 to 8 for two different choices of the subspaces $X_{n}$ : (a) $X_{n}=\bar{X}_{2 n}$, (b) $X_{n}=\bar{X}_{3 n}$.

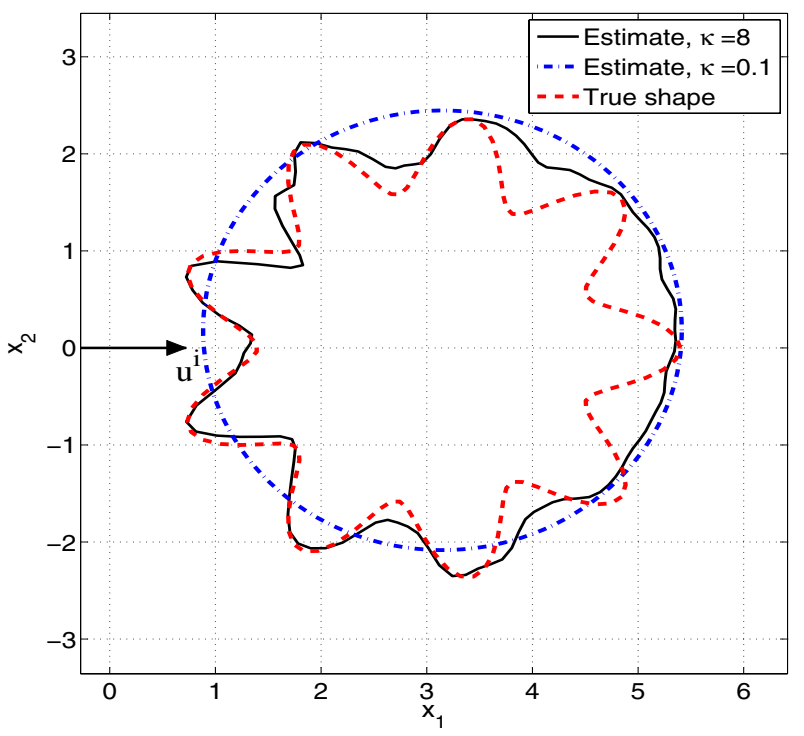

(a)

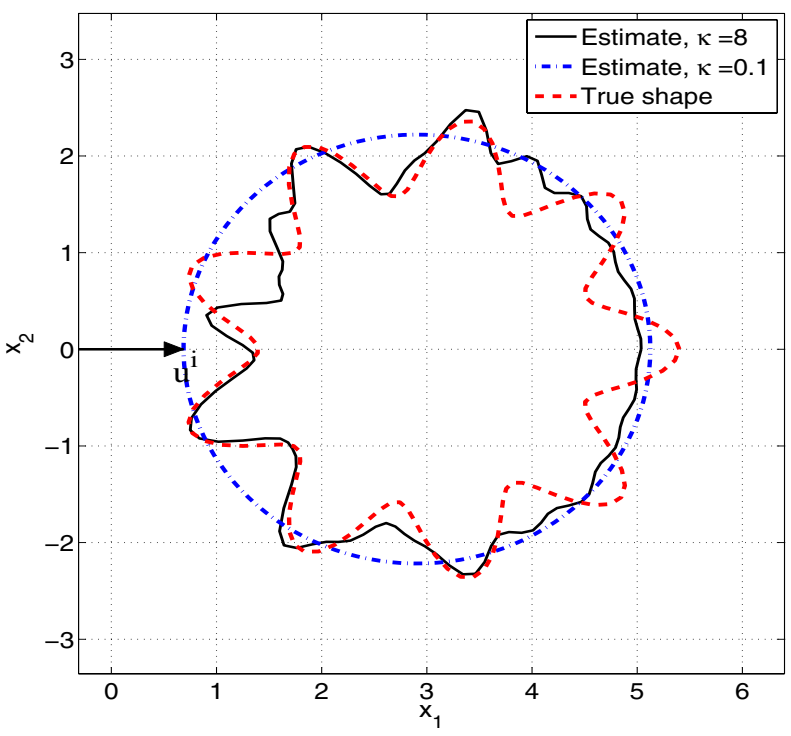

(b)

FiguRe 6. Reconstruction of obstacle 2 using 10 wavenumbers from 0.1 to 8 and 10 Newton iterations at each frequency: (a) $X_{n}=\bar{X}_{5 n}$, (b) $X_{n}=\bar{X}_{25}$ for all $n$. 


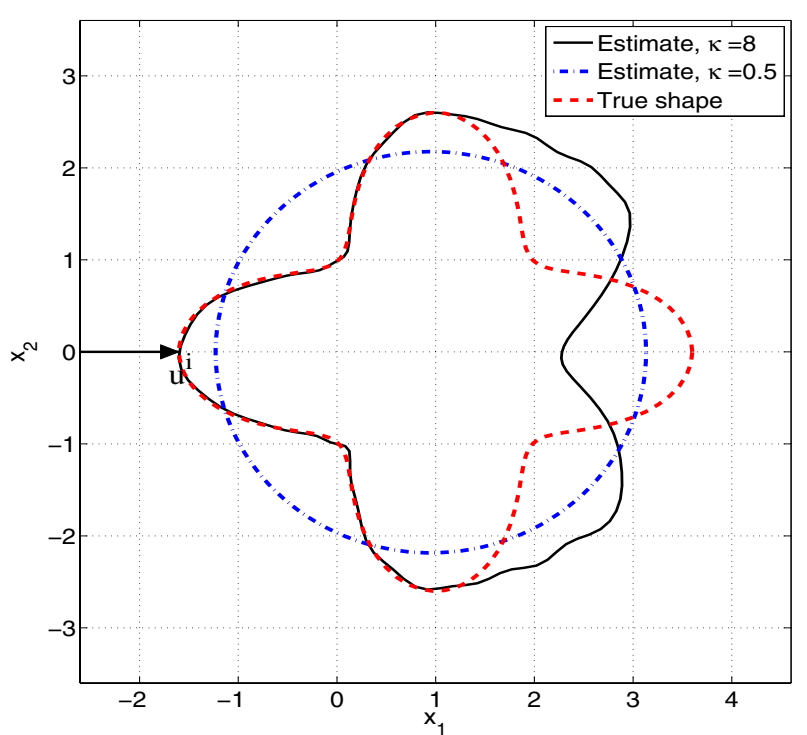

(a)

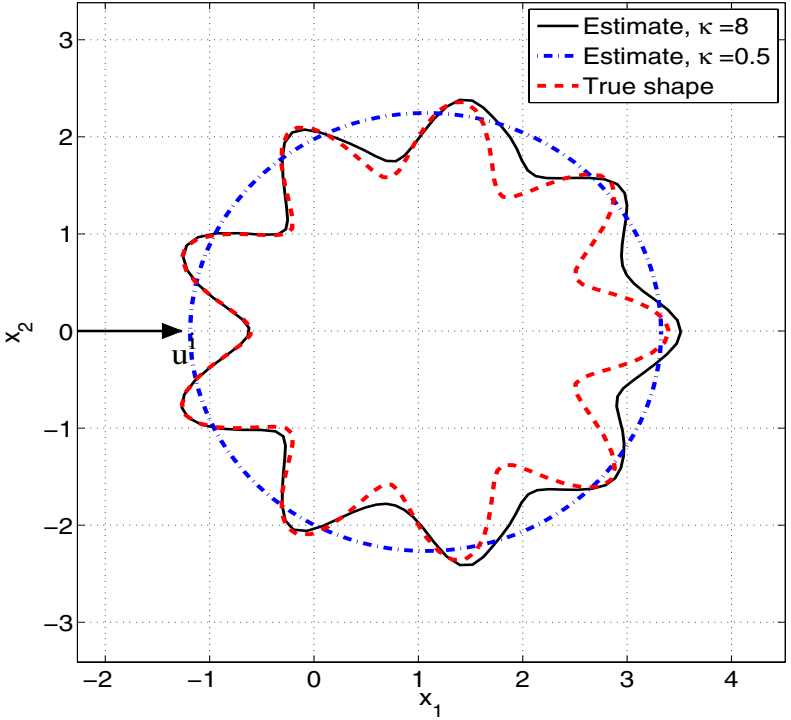

(b)

Figure 7. Reconstruction using the multi-level Newton method: (a) obstacle 1, (b) obstacle 2.

10 frequencies were chosen between 0.5 and 8 . The regularization parameter $\alpha$ at the first frequency step was chosen to be 0.04 which was 4 times larger than that at the other frequencies. Moreover, 15 iterations were used at the first step and 10 iterations were used at the other frequencies. We note that since the lowest frequency was chosen higher than in the previous tests, we chose $x^{0}=(1,0)$ for both obstacles and the initial guess of the center was chosen to be at $(-1,0)$. As can be seen, the reconstructions were comparable to Figure 3 which confirmed our theoretical analysis.

Acknowledgements. The authors are grateful to the anonymous referees for their valuable and stimulating comments and suggestions.

\section{REFERENCES}

[1] G. Alessandrini and L. Rondi, Determining a sound-soft polyhedral scatterer by a single far-field measurement. Proc. Amer. Math. Soc. 133 (2005) 1685-1691.

[2] H. Ammari, J. Garnier, H. Kang, M. Lim and K. Sølna, Multistatic imaging of extended targets. SIAM J. Imaging Sci. 5 (2012) 564-600.

[3] G. Bao and F. Triki, Error estimates for the recursive linearization of inverse medium problems. J. Comput. Math. 28 (2010) $725-744$.

[4] K. Belkebir, S. Bonnard, F. Pezin and P. Sabouroux and M. Saillard, Validation of 2D inverse scattering algorithms from multi-frequency experimental data. J. Electromagn. Waves Appl. 14 (2000) 1637-1667.

[5] S.N. Chandler-Wilde and P. Monk, Wave-number-explicit bounds in time-harmonic scattering. SIAM J. Math. Anal. 39 (2008) $1428-1455$.

[6] Y. Chen, Inverse scattering via Heisenberg's uncertainty principle. Inverse Probl. 13 (1997) 253-282.

[7] J. Cheng and M. Yamamoto, Global uniqueness in the inverse acoustic scattering problem within polygonal obstacles. Chin. Ann. Math. Ser. B 25 (2004) 1-6.

[8] W. Chew and J. Lin, A frequency-hopping approach for microwave imaging of large inhomogeneous bodies. IEEE Microwave Guided Wave Lett. 5 (1995) 439-441.

[9] D. Colton and A. Kirsch, A simple method for solving inverse scattering problems in the resonance region. Inverse Probl. 12 (1996) 383-393.

[10] D. Colton and R. Kress, Inverse acoustic and electromagnetic scattering theory, 3rd edn. Springer, New York, 2013. 
[11] D. Colton and B.D. Sleeman, Uniqueness theorems for the inverse problem of acoustic scattering. IMA J. Appl. Math. 31 (1983) 253-259.

[12] M.V. de Hoop, L. Qiu and O. Scherzer, A convergence analysis of a multi-level projected steepest descent iteration for nonlinear inverse problems in banach spaces subject to stability constraints. Preprint arXiv:1206.3706 [math.NA].

[13] G.B. Folland, Fourier analysis and its applications. The Wadsworth \& B Brooks/Cole Mathematics Series. Wadsworth \& Brooks/Cole Advanced Books \& Software, Pacific Grove, CA (1992).

[14] D. Gintides, Local uniqueness for the inverse scattering problem in acoustics via the Faber-Krahn inequality. Inverse Probl. 21 (2005) 1195-1205.

[15] R. Griesmaier, Multi-frequency orthogonality sampling for inverse obstacle scattering problems. Inverse Probl. 27 (2011) 085005.

[16] B.B. Guzina, F. Cakoni and C. Bellis, On the multi-frequency obstacle reconstruction via the linear sampling method. Inverse Probl. 26 (2010) 125005.

[17] N. Honda, G. Nakamura and M. Sini, Analytic extension and reconstruction of obstacles from few measurements for elliptic second order operators. Math. Annalen (2012). DOI: 10.1007/s00208-012-0786-0.

[18] V. Isakov, Inverse Problems for Partial Differential Equations, 2nd edn. Springer, New York (2006).

[19] A. Kirsch. The domain derivative and two applications in inverse scattering theory. Inverse Probl. 9 (1993) $81-96$.

[20] X. Liu and B. Zhang, Unique determination of a sound-soft ball by the modulus of a single far field datum. J. Math. Anal. Appl. (2010) 619-624.

[21] P.A. Martin, Multiple scattering. Interaction of time-harmonic waves with $N$ obstacles. In vol. 107 of Encycl. Math. Appl. Cambridge University Press, Cambridge (2006).

[22] W. McLean, Strongly elliptic systems and boundary integral equations. Cambridge University Press, Cambridge (2000).

[23] J.M. Melenk, Mapping properties of combined field Helmholtz boundary integral operators. SIAM J. Math. Anal. 44 (2012) 2599-2636.

[24] R. Potthast, Fréchet differentiability of boundary integral operators in inverse acoustic scattering. Inverse Probl. 10 (1994) 431-447.

[25] R. Potthast, A study on orthogonality sampling. Inverse Probl. 26 (2010) 074015.

[26] A.G. Ramm, Multidimensional inverse scattering problems. Longman Scientific \& Technical, Harlow (1992).

[27] E. Sincich and M. Sini, Local stability for soft obstacles by a single measurement. Inverse Probl. Imaging 2 (2008) 301-315.

[28] M. Sini and N.T. Thành, Inverse acoustic obstacle scattering problems using multifrequency measurements. Inverse Probl. Imaging 6 (2012) 749-773.

[29] P. Stefanov and G. Uhlmann, Local uniqueness for the fixed energy fixed angle inverse problem in obstacle scattering. Proc. Amer. Math. Soc. 132 (2004) 1351-1354 (electronic).

[30] A. Tijhuis, K. Belkebir, A. Litman and B. de Hon, Multi-frequency distorted-wave Born approach to 2D inverse profiling. Inverse Probl. 17 (2001) 1635-1644.

[31] A. Tijhuis, K. Belkebir, A. Litman and B. de Hon, Theoretical and computational aspects of 2-D inverse profiling. IEEE Trans. Geosci. Remote Sensing 39 (2001) 1316-1330. 